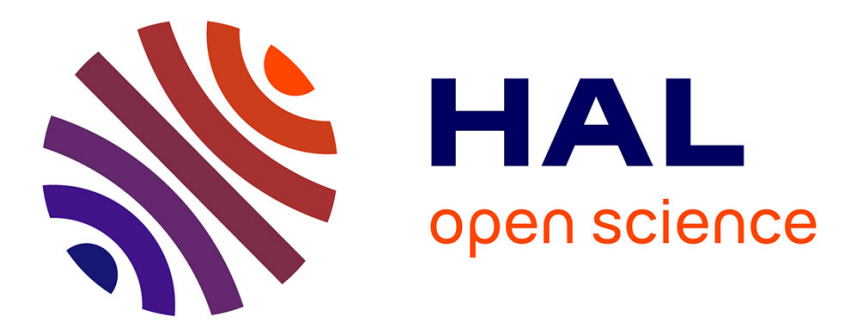

\title{
Linear and quadratic solid-shell finite elements SHB8PSE and SHB20E for the modeling of piezoelectric sandwich structures
}

Fessal Kpeky, Farid Abed-Meraim, Hakim Boudaoud, El Mostafa Daya

\section{- To cite this version:}

Fessal Kpeky, Farid Abed-Meraim, Hakim Boudaoud, El Mostafa Daya. Linear and quadratic solid-shell finite elements SHB8PSE and SHB20E for the modeling of piezoelectric sandwich structures. Mechanics of Advanced Materials and Structures, 2018, 25 (7), pp.559-578. 10.1080/15376494.2017.1285466 . hal-02389489

\section{HAL Id: hal-02389489 \\ https://hal.science/hal-02389489}

Submitted on 2 Dec 2019

HAL is a multi-disciplinary open access archive for the deposit and dissemination of scientific research documents, whether they are published or not. The documents may come from teaching and research institutions in France or abroad, or from public or private research centers.
L'archive ouverte pluridisciplinaire HAL, est destinée au dépôt et à la diffusion de documents scientifiques de niveau recherche, publiés ou non, émanant des établissements d'enseignement et de recherche français ou étrangers, des laboratoires publics ou privés. 


\title{
Linear and quadratic solid-shell finite elements SHB8PSE and SHB20E for the modeling of piezoelectric sandwich structures
}

\author{
Fessal Kpeky $^{1}$, Farid Abed-Meraim ${ }^{2,3^{*}}$, Hakim Boudaoud ${ }^{1}$ and El Mostafa Daya ${ }^{1,3}$ \\ ${ }^{1}$ LEM3, UMR CNRS 7239, Université de Lorraine, Ile du Saulcy Metz Cedex 01, France \\ ${ }^{2}$ LEM3, UMR CNRS 7239, Arts et Métiers ParisTech, 4 rue Augustin Fresnel, 57078 \\ Metz Cedex 03, France \\ ${ }^{3}$ Laboratory of Excellence on Design of Alloy Metals for low-mAss Structures (DAMAS), \\ France
}

\begin{abstract}
In this paper, hexahedral piezoelectric solid-shell finite element formulations, with linear and quadratic interpolation, denoted by SHB8PSE and SHB20E, respectively, are proposed for the modeling of piezoelectric sandwich structures. Compared to conventional solid and shell elements, the solid-shell concept reveals to be very attractive, due to a number of well-established advantages and computational capabilities. More specifically, the present study is devoted to the modeling and analysis of multilayer structures that incorporate piezoelectric materials in the form of layers or patches. The interest in this solid-shell approach is shown through a set of selective and representative benchmark problems. These include numerical tests applied to various configurations of beam, plate and shell structures, both in static and vibration analysis. The results yielded by the proposed formulations are compared with those given by state-of-the-art piezoelectric elements available in ABAQUS; in particular, the C3D20E quadratic hexahedral finite element with piezoelectric degrees of freedom.
\end{abstract}

Keywords: Finite elements, Solid-shell, Piezoelectric effect, Sandwich structures, Vibration analysis.

\section{Introduction}

With the ever growing technological developments, smart combination of key properties of materials is being advantageously used in many engineering fields. Examples of these are the multilayer structures that combine elastic, viscoelastic and piezoelectric layers, which are increasingly used in civil engineering, automotive and aerospace as well as in bioengineering. Shape control and vibration damping are some of the major applications of these materials.

Over the past few decades, considerable attention has been devoted to thin structures that combine piezoelectric material layers or patches, due to their many potential applications. The 
resulting smart materials and structures are used nowadays in vibration control [1-3], shape control [4-6], noise and acoustic control [7-10] as well as health monitoring of civil infrastructures [11-13]. Predicting the behavior of such materials and structures is therefore crucial for their proper implementation. To this end, the numerical simulation, e.g. by means of the finite element method, represents a very convenient and powerful approach, especially due to its very reasonable cost and its flexibility. Since the early work of Allik and Hughes [14], several tools have been proposed in the literature to model piezoelectric structures. Reviews on mechanical models and finite element formulations, which can be found in [1518], reveal that a large variety of $2 \mathrm{D}$ and $3 \mathrm{D}$ piezoelectric finite elements have actually been developed.

Han and Lee [19] and Hwang and Park [20] extended the Classical Laminate Plate Theory (CLPT) presented in [21] to the analysis of composite plates with piezoelectric actuators using 2D finite elements based on Kirchhoff's assumptions. Other contributors, such as [22-24] and [25-28], used First-order Shear Deformation Theory (FSDT) and Higher-order Shear Deformation Theory (HSDT), respectively. To enrich the kinematics, Kapuria et al. [29-32] introduced in the above works the well-known zigzag theory. Interesting contributions to the field were also made by Boudaoud et al. [33], Belouettar et al. [3] and Azrar et al. [34], among others. Moreover, the Carrera Unified Formulation (CUF) models for piezoelectric plates were proposed in Ballhause et al. [35] as well as in Robaldo et al. [36]. In these works, both equivalent single layer (ESL) and layer-wise (LW) assumptions were considered and many tests were successfully conducted in static and dynamic on laminated plates embedding piezoelectric layers. Several other contributions based on this approach can also be found in [37-41]. All of these formulations are capable of efficiently modeling beam and plate structures comprising piezoelectric materials. However, in real-life applications, it is quite common that relatively thin components coexist with thick structures, such as very thin piezoelectric patch sensors used for the monitoring of civil infrastructures. Therefore, the accurate and efficient modeling of such structures has motivated the development of new finite element technologies, among which the solid-shell concept. In this context also, several finite element models of this type have been proposed in the literature (see, e.g., [2, 42-46]). In particular, Sze et al. [42, 43] proposed hybrid finite element modeling of smart structures. In their work, the variation of electric potential was assumed to be linear along the thickness. Their formulation was subsequently extended by Zheng et al. [46] to the refined hybrid element. Alternatively, Klinkel and Wagner [44, 45] assumed in their contributions a quadratic distribution for the electric potential across the thickness. The geometric nonlinearities were taken into account, but application of their model was restricted to structures combining elastic and piezoelectric layers. Tan and Vu-Quoc [2] also successfully modeled piezoelectric beam and plate structures under static and vibration conditions. More recently, Kulikov and Plotnikova [47, 48] developed solid-shell finite elements, which are like most of those developed in the literature, namely having a 2D geometry, while allowing a 3D constitutive law to be considered.

Despite the availability of the above-discussed models in the literature, it appears that so far, commercial finite element software packages, such as ABAQUS and ANSYS, only 
propose solid piezoelectric finite element technologies. The latter are known to be quite expensive in terms of CPU time in the modeling of thin structures, which significantly reduces their efficiency. It must be noted, however, that several studies in the literature, such as [49-51], have proposed piezoelectric shell elements that have been implemented using ABAQUS User Element (UEL) subroutine.

The current contribution proposes extension of the SHB8PS and SHB20 linear and quadratic solid-shell elements, respectively presented in [52-54] and [55], to the modeling of structures that contain piezoelectric materials. The paper is organized as follows. In Section 2, the coupled electromechanical constitutive equations are presented as well as the discretized problem to be solved by the finite element method. Section 3 details the formulation of the SHB8PSE and SHB20E piezoelectric solid-shell elements, which are based on linear and quadratic interpolation, respectively. To assess the performance of the proposed piezoelectric solid-shell elements and for validation purposes, a set of selective and representative benchmark tests are conducted in Section 4, both in static and vibration analysis. Finally, Section 5 summarizes the main contributions along with some concluding remarks.

\section{Constitutive equations and discretization of the problem}

\subsection{Coupled electromechanical constitutive equations}

Piezoelectric materials have the capability of generating electricity when subjected to mechanical loading (sensors). Conversely, they also have the ability to deform under electrical charging (actuators). These properties are described by the following coupled electromechanical equations:

$$
\left\{\begin{array}{l}
\boldsymbol{\sigma}=\mathbf{C} \cdot \boldsymbol{\varepsilon}-\mathbf{e}^{T} \cdot \boldsymbol{E} \\
\boldsymbol{D}=\mathbf{e} \cdot \boldsymbol{\varepsilon}+\mathbf{K} \cdot \boldsymbol{E}
\end{array}\right.
$$

where $\boldsymbol{\sigma}$ and $\boldsymbol{\varepsilon}$ represent, respectively, the vector form of the stress and strain tensors; $\boldsymbol{D}$ and $\boldsymbol{E}$ denote the electric displacement and electric field vector, respectively; while $\mathbf{C}, \mathbf{e}$ and $\mathbf{\kappa}$ stand for the elastic, piezoelectric and dielectric permittivity matrix, respectively.

The discretized forms $\{\boldsymbol{\varepsilon}\}$ and $\{\boldsymbol{E}\}$ for the strain tensor and the electric field vector are related, respectively, to the discretized displacement $\{\boldsymbol{u}\}$ and to the discretized electric potential $\{\phi\}$, using the discrete gradient operators $\left[\mathbf{B}^{u}\right]$ and $\left[\mathbf{B}^{\phi}\right]$, as follows:

$$
\left\{\begin{array}{l}
\{\boldsymbol{\varepsilon}\}=\left[\mathbf{B}^{u}\right]\{\boldsymbol{u}\} \\
\{\boldsymbol{E}\}=-\left[\mathbf{B}^{\phi}\right]\{\boldsymbol{\phi}\}
\end{array}\right.
$$


In the current contribution, the discrete gradient operators $\left[\mathbf{B}^{u}\right]$ and $\left[\mathbf{B}^{\phi}\right]$ are obtained by finite element discretization for each of the proposed piezoelectric solid-shell formulations SHB8PSE and SHB20E, as will be detailed in Section 3.

\subsection{Discretized problem}

The variational principle pertaining to piezoelectric materials, which provides the governing equations for the associated boundary value problem, is described by the Hamilton principle [14]. In this weak form of equations of motion, the Lagrangian and the virtual work are appropriately adapted to include the electrical contributions, in addition to the more classical mechanical fields

$$
\begin{aligned}
& -\int_{V} \rho \ddot{\boldsymbol{u}} \cdot \delta \boldsymbol{u} d v-\int_{V} \boldsymbol{\sigma} \cdot \delta \boldsymbol{\varepsilon} d v+\int_{V} \boldsymbol{f}_{v} \cdot \delta \boldsymbol{u} d v+\int_{S} \boldsymbol{f}_{s} \cdot \delta \boldsymbol{u} d s+\boldsymbol{f}_{p} \cdot \delta \boldsymbol{u} \\
& =-\int_{V} \boldsymbol{D} \cdot \boldsymbol{\delta} \boldsymbol{E} d v+\int_{V} \boldsymbol{q}_{v} \cdot \delta \phi d v+\int_{S} \boldsymbol{q}_{s} \cdot \delta \boldsymbol{\phi} d s+\boldsymbol{q}_{p} \cdot \delta \boldsymbol{\phi}
\end{aligned}
$$

where $\rho$ is the material density; $\boldsymbol{q}_{v}, \boldsymbol{q}_{s}$ and $\boldsymbol{q}_{p}$ denote volume, surface and point charge, respectively; while $\boldsymbol{f}_{v}, \boldsymbol{f}_{s}$ and $\boldsymbol{f}_{p}$ represent volume, surface and point force, respectively.

The finite element discretization of the boundary value problem governed by Eq. (3) generally leads to the following system of discretized equations:

$$
\left\{\begin{array}{r}
{\left[\mathbf{M}^{u u}\right]\{\ddot{\boldsymbol{U}}\}+\left[\mathbf{K}^{u u}\right]\{\boldsymbol{U}\}+\left[\mathbf{K}^{u \phi}\right]\{\boldsymbol{\phi}\}=\{\boldsymbol{F}\}} \\
{\left[\mathbf{K}^{\phi u}\right]\{\boldsymbol{U}\}+\left[\mathbf{K}^{\phi \phi}\right]\{\boldsymbol{\phi}\}=\{\boldsymbol{Q}\}}
\end{array}\right.
$$

where all matrices and vectors involved in Eq. (4) are explicitly defined in Table 1.

\section{Formulation of linear and quadratic piezoelectric solid-shell finite elements}

The proposed hexahedral piezoelectric solid-shell finite elements SHB8PSE and SHB20E are extensions of the linear and quadratic solid-shell elements SHB8PS and SHB20, which were developed in [52, 53] and [55], respectively, based on purely mechanical modeling. The starting point for these piezoelectric extensions is the addition of one piezoelectric degree of freedom to each node of their mechanical finite element counterparts. The outline of these formulations is given in the following sections.

\subsection{Kinematics and interpolation}

The piezoelectric solid-shell elements SHB8PSE and SHB20E denote an eight-node hexahedral element and a twenty-node one, respectively. These elements have at each of their nodes three displacement degrees of freedom as well as one electric degree of freedom. Similar to their mechanical counterparts SHB8PS and SHB20, a special direction is chosen, 
designated as the "thickness", normal to the mean plane of these elements. Also, an in-plane reduced-integration rule is adopted, with $1 \times n_{\text {int }}$ integration points for the SHB8PSE element and $4 \times n_{\text {int }}$ for the SHB20E (see, e.g., Fig. 1, in the particular case of $n_{\text {int }}=5$ ).

For the SHB8PSE and SHB20E elements, the spatial coordinates $x_{i}$ are related to the nodal coordinates $x_{i I}$ using linear and quadratic shape functions, respectively, as follows:

$$
x_{i}=x_{i I} N_{I}(\xi, \eta, \zeta)
$$

where $i$ represents the spatial directions and ranges from 1 to 3 ; while $I$ stands for the node number, which ranges from 1 to 8 , for the SHB8PSE element, and from 1 to 20 for the SHB20E. Likewise, the displacement field $u_{i}$ and potential field $\phi$ are related to the nodal displacements $u_{i I}$ and nodal potentials $\phi_{I}$, respectively, using the shape functions

$$
\left\{\begin{array}{l}
u_{i}=u_{i I} N_{I}^{u}(\xi, \eta, \zeta) \\
\phi=\phi_{I} N_{I}^{\phi}(\xi, \eta, \zeta)
\end{array}\right.
$$

Note that in Eqs. (5) and (6) above, the convention of implied summation over the repeated index $I$ has been adopted.

\subsection{Discrete gradient operators}

For both elements SHB8PSE and SHB20E, the corresponding discrete gradient operators $\left[\mathbf{B}^{u}\right]$ and $\left[\mathbf{B}^{\phi}\right]$ can be derived in the following compact form:

$$
\mathbf{B}^{u}=\left[\begin{array}{ccc}
\boldsymbol{b}_{1}^{T}+h_{\alpha, 1} \boldsymbol{\gamma}_{\alpha}^{T} & \mathbf{0} & \mathbf{0} \\
\mathbf{0} & \boldsymbol{b}_{2}^{T}+h_{\alpha, 2} \boldsymbol{\gamma}_{\alpha}^{T} & \mathbf{0} \\
\mathbf{0} & \mathbf{0} & \boldsymbol{b}_{3}^{T}+h_{\alpha, 3} \boldsymbol{\gamma}_{\alpha}^{T} \\
\boldsymbol{b}_{2}^{T}+h_{\alpha, 2} \boldsymbol{\gamma}_{\alpha}^{T} & \boldsymbol{b}_{1}^{T}+h_{\alpha, 1} \boldsymbol{\gamma}_{\alpha}^{T} & \mathbf{0} \\
\boldsymbol{b}_{3}^{T}+h_{\alpha, 3} \boldsymbol{\gamma}_{\alpha}^{T} & \mathbf{0} & \boldsymbol{b}_{1}^{T}+h_{\alpha, 1} \boldsymbol{\gamma}_{\alpha}^{T} \\
\mathbf{0} & \boldsymbol{b}_{3}^{T}+h_{\alpha, 3} \boldsymbol{\gamma}_{\alpha}^{T} & \boldsymbol{b}_{2}^{T}+h_{\alpha, 2} \boldsymbol{\gamma}_{\alpha}^{T}
\end{array}\right] \quad ; \quad \mathbf{B}^{\phi}=\left[\begin{array}{l}
\boldsymbol{b}_{1}^{T}+h_{\alpha, 1} \gamma_{\alpha}^{T} \\
\boldsymbol{b}_{2}^{T}+h_{\alpha, 2} \boldsymbol{\gamma}_{\alpha}^{T} \\
\boldsymbol{b}_{3}^{T}+h_{\alpha, 3} \gamma_{\alpha}^{T}
\end{array}\right]
$$

where $\boldsymbol{b}_{j}^{T}, h_{\alpha, j}$ and $\boldsymbol{\gamma}_{\alpha}^{T}$ are detailed in Appendix A. More details can also be found in references [52-55]. Note again that, in Eq. (7) and in what follows, the convention of implied summation over the repeated index $\alpha$ is adopted, with $\alpha$ ranging from 1 to 4 , for the SHB8PSE element, and from 1 to 16 for the SHB20E.

Similar to the purely mechanics-based solid-shell element SHB20 (see, e.g., [55]), the benchmark tests performed with the piezoelectric solid-shell counterpart SHB20E did not reveal any particular locking and, accordingly, no specific enhanced assumed strain 
techniques have been applied to this quadratic solid-shell element. By contrast, the original formulation of the linear solid-shell version SHB8PSE has been shown to suffer both from spurious hourglass modes and locking phenomena. Therefore, to circumvent these numerical pathologies, projection of its discrete gradient operator and stabilization of its stiffness matrix are undertaken following the assumed strain method. Note that the projection of the displacement-related discrete gradient operator $\mathbf{B}^{u}$ is performed in the same way as for the SHB8PS element (see [53]), which leads to a similar form for the stiffness matrix $\mathbf{K}^{u u}$. Hence, in the current work, special attention is paid to the impact of the assumed-strain projection on the expressions of the piezoelectric and dielectric matrices $\mathbf{K}^{u \phi}$ and $\mathbf{K}^{\phi \phi}$.

\subsection{Assumed-strain projection and stabilization of the SHBSPSE}

Let us first underline that in Eq. (7), vectors $\boldsymbol{b}_{i}$ originally defined by Hallquist's form are replaced by the following mean form proposed by Flanagan and Belytschko [56]:

$$
\hat{\boldsymbol{b}}_{i}=\frac{1}{V_{e}} \int_{V_{e}} \boldsymbol{N}_{, i}^{u}(\xi, \eta, \zeta) d v
$$

Then, using the $\mathrm{Hu}-\mathrm{Washizu}$ mixed variational principle in conjunction with the assumed strain method proposed by Belytschko and Bindeman [57], the discrete gradient operator $\hat{\mathbf{B}}^{u}$ is projected onto a new operator $\overline{\hat{\mathbf{B}}}^{u}$ in order to eliminate various types of locking. The latter operator is additively decomposed into two parts, denoted by $\hat{\mathbf{B}}_{12}^{u}$ and $\overline{\hat{\mathbf{B}}}_{34}^{u}$, as follows:

$$
\left[\hat{\mathbf{B}}_{12}^{u}\right]=\left[\begin{array}{ccc}
\hat{\boldsymbol{b}}_{1}^{T}+\hat{\boldsymbol{X}}_{12}^{T} & \mathbf{0} & \mathbf{0} \\
\mathbf{0} & \hat{\boldsymbol{b}}_{2}^{T}+\hat{\boldsymbol{Y}}_{12}^{T} & \mathbf{0} \\
\mathbf{0} & \mathbf{0} & \hat{\boldsymbol{b}}_{3}^{T}+\hat{\boldsymbol{Z}}_{12}^{T} \\
\hat{\boldsymbol{b}}_{2}^{T}+\hat{\boldsymbol{Y}}_{12}^{T} & \hat{\boldsymbol{b}}_{1}^{T}+\hat{\boldsymbol{X}}_{12}^{T} & \mathbf{0} \\
\hat{\boldsymbol{b}}_{3}^{T}+\hat{\boldsymbol{Z}}_{12}^{T} & \mathbf{0} & \hat{\boldsymbol{b}}_{1}^{T}+\hat{\boldsymbol{X}}_{12}^{T} \\
\mathbf{0} & \hat{\boldsymbol{b}}_{3}^{T}+\hat{\boldsymbol{Z}}_{12}^{T} & \hat{\boldsymbol{b}}_{2}^{T}+\hat{\boldsymbol{Y}}_{12}^{T}
\end{array}\right] \quad ;\left[\begin{array}{ccc}
\hat{\boldsymbol{X}}_{34}^{T} & \mathbf{0} & \mathbf{0} \\
\mathbf{0} & \hat{\boldsymbol{Y}}_{34}^{T} & \mathbf{0} \\
\mathbf{0} & \mathbf{0} & \hat{\boldsymbol{Z}}_{3}^{T} \\
\mathbf{0} & \mathbf{0} & \mathbf{0} \\
\mathbf{0} & \mathbf{0} & \mathbf{0} \\
\mathbf{0} & \mathbf{0} & \mathbf{0}
\end{array}\right]=\left[\begin{array}{c} 
\\
\end{array}\right.
$$

where

$$
\hat{\boldsymbol{X}}_{\beta \gamma}=\sum_{\alpha=\beta}^{\gamma} h_{\alpha, x} \hat{\gamma}_{\alpha} \quad ; \quad \hat{\boldsymbol{Y}}_{\beta \gamma}=\sum_{\alpha=\beta}^{\gamma} h_{\alpha, y} \hat{\gamma}_{\alpha} \quad ; \quad \hat{\boldsymbol{Z}}_{\beta \gamma}=\sum_{\alpha=\beta}^{\gamma} h_{\alpha, z} \hat{\gamma}_{\alpha}
$$

The stiffness matrix is finally derived as follows:

$$
\mathbf{K}_{e}^{u u}=\mathbf{K}_{12}^{u u}+\mathbf{K}^{u u}{ }_{\text {Stab }}
$$

where 


$$
\begin{aligned}
& \mathbf{K}^{u u}{ }_{12}=\int_{V_{e}} \hat{\mathbf{B}}_{12}^{u T} \cdot \mathbf{C} \cdot \hat{\mathbf{B}}_{12}^{u} d v \\
& \mathbf{K}_{S t a b}^{u u}=\int_{V_{e}} \hat{\mathbf{B}}_{12}^{u T} \cdot \mathbf{C} \cdot \overline{\hat{\mathbf{B}}}_{34}^{u} d v+\int_{V_{e}} \overline{\hat{\mathbf{B}}}_{34}^{u T} \cdot \mathbf{C} \cdot \hat{\mathbf{B}}_{12}^{u} d v+\int_{V_{e}} \overline{\hat{\mathbf{B}}}_{34}^{u T} \cdot \mathbf{C} \cdot \overline{\hat{\mathbf{B}}}_{34}^{u} d v
\end{aligned}
$$

The stabilization stiffness matrix $\mathbf{K}^{u u}{ }_{\text {Stab }}$ is computed in a co-rotational coordinate frame. The adopted orthogonal co-rotational coordinate system is defined by the rotation matrix $\boldsymbol{R}$ that maps a vector in the global coordinate system to the co-rotational one. The components of the first two column vectors of matrix $\boldsymbol{R}$, denoted $a_{1 i}$ and $a_{2 i}$, respectively, are given by

$$
\begin{aligned}
& a_{1 i}=\boldsymbol{\Lambda}_{1}^{T} \cdot \boldsymbol{x}_{i}, \quad a_{2 i}=\boldsymbol{\Lambda}_{2}^{T} \cdot \boldsymbol{x}_{i}, \quad i=1,2,3 \\
& \text { with }\left\{\begin{array}{l}
\boldsymbol{\Lambda}_{1}^{T}=(-1,1,1,-1,-1,1,1,-1) \\
\boldsymbol{\Lambda}_{2}^{T}=(-1,-1,1,1,-1,-1,1,1)
\end{array}\right.
\end{aligned}
$$

Then, the second column vector $\boldsymbol{a}_{2}$ is modified in order to make it orthogonal to $\boldsymbol{a}_{1}$. To this end, a correction vector $\boldsymbol{a}_{c}$ is added to $\boldsymbol{a}_{2}$ such that:

$$
\boldsymbol{a}_{1}^{T} \cdot\left(\boldsymbol{a}_{2}+\boldsymbol{a}_{c}\right)=0 \quad \Rightarrow \quad \boldsymbol{a}_{c}=-\frac{\boldsymbol{a}_{1}^{T} \cdot \boldsymbol{a}_{2}}{\boldsymbol{a}_{1}^{T} \cdot \boldsymbol{a}_{1}} \boldsymbol{a}_{1}
$$

The third base vector $\boldsymbol{a}_{3}$ is finally obtained by the cross-product $\boldsymbol{a}_{3}=\boldsymbol{a}_{1} \wedge\left(\boldsymbol{a}_{2}+\boldsymbol{a}_{c}\right)$, which gives the components of the rotation matrix after normalization by

$$
R_{1 i}=\frac{a_{1 i}}{\left\|\boldsymbol{a}_{1}\right\|} \quad, \quad R_{2 i}=\frac{a_{2 i}+a_{c i}}{\left\|\boldsymbol{a}_{2}+\boldsymbol{a}_{c}\right\|} \quad, \quad R_{3 i}=\frac{a_{3 i}}{\left\|\boldsymbol{a}_{3}\right\|} \quad, \quad i=1,2,3
$$

For the derivation of the piezoelectric and dielectric matrices $\mathbf{K}^{u \phi}$ and $\mathbf{K}^{\phi \phi}$, a similar procedure is followed. As above, the potential-related discrete gradient operator $\hat{\mathbf{B}}^{\phi}$ is projected onto $\overline{\mathbf{B}}^{\phi}$, which is then additively decomposed into two parts, denoted by $\hat{\mathbf{B}}_{12}^{\phi}$ and $\overline{\hat{\mathbf{B}}}_{34}^{\phi}$, as follows:

$$
\hat{\mathbf{B}}_{12}^{\phi}=\left[\begin{array}{c}
\hat{\boldsymbol{b}}_{1}^{T}+\hat{\boldsymbol{X}}_{12}^{T} \\
\hat{\boldsymbol{b}}_{2}^{T}+\hat{\boldsymbol{Y}}_{12}^{T} \\
\hat{\boldsymbol{b}}_{3}^{T}+\hat{\boldsymbol{Z}}_{12}^{T}
\end{array}\right] \quad ; \quad \overline{\hat{\mathbf{B}}}_{34}^{\phi}=\left[\begin{array}{c}
\hat{\boldsymbol{X}}_{34}^{T} \\
\hat{\boldsymbol{Y}}_{34}^{T} \\
\hat{\boldsymbol{Z}}_{3}^{T}
\end{array}\right]
$$

Accordingly, the piezoelectric matrix is computed as follows:

$$
\mathbf{K}^{u \phi}{ }_{e}=\mathbf{K}_{12}^{u \phi}+\mathbf{K}^{u \phi}{ }_{S t a b}
$$

with 


$$
\begin{aligned}
& \mathbf{K}^{u \phi}{ }_{12}=\int_{V_{e}} \hat{\mathbf{B}}_{12}^{u T} \cdot \mathbf{e}^{T} \cdot \hat{\mathbf{B}}_{12}^{\phi} d v \\
& \mathbf{K}^{u \phi}{ }_{S t a b}=\int_{V_{e}} \hat{\mathbf{B}}_{12}^{u T} \cdot \mathbf{e}^{T} \cdot \overline{\hat{\mathbf{B}}}_{34}^{\phi} d v+\int_{V_{e}} \overline{\hat{\mathbf{B}}}_{34}^{u T} \cdot \mathbf{e}^{T} \cdot \hat{\mathbf{B}}_{12}^{\phi} d v+\int_{V_{e}} \overline{\hat{\mathbf{B}}}_{34}^{u T} \cdot \mathbf{e}^{T} \cdot \overline{\hat{\mathbf{B}}}_{34}^{\phi} d v
\end{aligned}
$$

while the dielectric matrix is given by

$$
\mathbf{K}_{e}^{\phi \phi}=\mathbf{K}_{12}^{\phi \phi}+\mathbf{K}^{\phi \phi}{ }_{S t a b}
$$

with

$$
\begin{aligned}
& \mathbf{K}_{12}^{\phi \phi}=-\int_{V_{e}} \hat{\mathbf{B}}_{12}^{\phi T} \cdot \mathbf{K} \cdot \hat{\mathbf{B}}_{12}^{\phi} d v \\
& \mathbf{K}_{\text {Stab }}^{\phi \phi}=-\int_{V_{e}} \hat{\mathbf{B}}_{12}^{\phi T} \cdot \mathbf{K} \cdot \overline{\hat{\mathbf{B}}}_{34}^{\phi} d v-\int_{V_{e}} \overline{\hat{\mathbf{B}}}_{34}^{\phi T} \cdot \mathbf{\kappa} \cdot \hat{\mathbf{B}}_{12}^{\phi} d v-\int_{V_{e}} \overline{\hat{\mathbf{B}}}_{34}^{\phi T} \cdot \mathbf{\kappa} \cdot \overline{\hat{\mathbf{B}}}_{34}^{\phi} d v
\end{aligned}
$$

Note that the stabilization matrices $\mathbf{K}^{u \phi}$ Stab and $\mathbf{K}_{\text {Stab }}^{\phi \phi}$, related to the piezoelectric and dielectric matrices, are computed in the same co-rotational coordinate frame as that used previously for the computation of the stabilization stiffness matrix. More details can be found in Appendix B.

Moreover, it must be noted that the material properties of the piezoelectric components, which are defined by the elastic, piezoelectric and dielectric permittivity matrices $\mathbf{C}, \mathbf{e}$ and $\boldsymbol{\kappa}$, are expressed in a local physical material frame. Hence, to express these matrices in the global coordinate system, an inverse transformation is used, which is defined by a rotation matrix, denoted $\boldsymbol{R}$. More details on these coordinate system transformations and on the derivation of the associated matrix components are given in Appendix C.

\section{Numerical tests and validation}

To evaluate the performance of the proposed piezoelectric solid-shell formulations, a selection of representative benchmark tests is conducted in static and vibration analysis, for multilayer beam, plate and shell structures. For all of the simulations, the mesh nomenclature adopted for hexahedral elements is as follows: $\left(N_{1} \times N_{2} \times N_{3}\right)$ elements, where $N_{1}$ denotes the number of elements in the length direction, $N_{2}$ is the number of elements in the width direction, and $N_{3}$ is the number of elements in the thickness direction. Note that, for the proposed solid-shell elements SHB8PSE and SHB20E, two integration points along the thickness direction are sufficient for the following computations, as the corresponding benchmark tests do not involve material nonlinearities. However, it must be noted that, when nonlinear material behavior models enter into play, more through-thickness integration points are required (for instance, five through-thickness integration points are recommended when elasto-plastic constitutive models are used, see, e.g., reference [53]). 


\subsection{Static benchmarks}

In the case of static analysis, the system of discretized equations (4) reduces to

$$
\left[\begin{array}{ll}
\mathbf{K}^{u u} & \mathbf{K}^{u \phi} \\
\mathbf{K}^{\phi u} & \mathbf{K}^{\phi \phi}
\end{array}\right]\left\{\begin{array}{l}
\boldsymbol{U} \\
\boldsymbol{\phi}
\end{array}\right\}=\left\{\begin{array}{l}
\boldsymbol{F} \\
\boldsymbol{Q}
\end{array}\right\}
$$

A set of five benchmark tests taken from the literature is investigated in the following sections. The first and third tests are devoted to beam structures (bimorph and sandwich beam in extension and shear deformation mechanisms, respectively). The second and fourth tests are dedicated to plate structures (investigation of shear locking in thin piezoelectric sensors and shape control of plate with piezoelectric patches, respectively). Finally, the fifth test deals with a piezoelectric sandwich shell structure.

\subsubsection{Bimorph pointer}

This benchmark test consists of a cantilever beam composed of two layers polarized in two opposite directions ( $\mathrm{z}$ and $-\mathrm{z}$ here). This test has been considered in many works in the literature, among which Tzou and Ye [58], Sze et al. [43] and Klinkel and Wagner [44]. A voltage of $\Delta \phi=1 \mathrm{~V}$ is applied on the exterior faces. The geometric dimensions and boundary conditions are reported in Fig. 2.

The material parameters are reported in Table 2, where the results obtained with the proposed piezoelectric solid-shell elements SHB8PSE and SHB20E are compared with those given by their counterparts from ABAQUS, which have the same geometry and the same number of degrees of freedom, namely the three-dimensional linear and quadratic elements C3D8E and C3D20E, respectively. These simulation results are additionally compared to the reference solution given by the following analytical formula:

$$
U_{z}(x)=-\frac{3}{2} \frac{\mathrm{e}_{31} \Delta \phi}{E h^{2}} x^{2}
$$

It appears from Table 2 that the proposed piezoelectric solid-shell element SHB8PSE provides a more accurate result than the standard three-dimensional ABAQUS element C3D8E, while requiring much less degrees of freedom. As to the quadratic versions of these elements, the accuracy of the proposed piezoelectric solid-shell element SHB20E is comparable to that of the three-dimensional ABAQUS element C3D20E, while using twice less elements. It is also noteworthy that despite the higher number of elements required for the C3D8E (40 times more elements than the SHB8PSE), its result still exhibits an error of nearly $26 \%$ with respect to the reference solution.

In the previous configuration of regular meshes, the higher performance of the proposed solid-shell elements has been clearly shown. In particular, fewer elements are generally required with the proposed solid-shell formulations to achieve convergence, as compared to their ABAQUS counterparts, which allows reducing the computational effort. This superiority 
in terms of higher convergence rate is even more evident in presence of distorted meshes. To illustrate this, we consider again the previous piezoelectric bimorph, which is discretized now with distorted meshes as shown in Fig. 3. Two different discretizations are considered for each element, and we investigate the sensitivity of the results to the corresponding distorted meshes. The normalized tip displacement is plotted in Fig. 4 for different values of the distortion parameter $r=2 e / a$, where $a$ is the in-plane element size and $e$ defines the twist with respect to the regular mesh. For the same mesh discretization of $10 \times 1 \times 2$ elements, the results reported in Fig. 4-b show that the relative error for the C3D20E element increases to $50 \%$ at a distortion parameter of $r=3$, when it is only $8 \%$ for the SHB20E at the same distortion. Also, with mesh refinement, the relative error decreases to about $5 \%$ for the C3D20E element, while it tends to zero for the SHB20E element. Regarding the linear elements, Fig. 4-a reveals that the relative error for the C3D8E element exceeds 20\%, at a distortion parameter of $r=3$, for both of the distorted meshes considered. However, although the SHB8PSE exhibits more sensitivity to mesh distortion than its quadratic counterpart SHB20E, its accuracy is still much better than that of the C3D8E element. This example clearly shows the interest of using solid-shell finite element technology to model piezoelectric structures, which is even more evident in presence of distorted meshes.

\subsubsection{Shear locking in thin piezoelectric sensors}

The aim of this benchmark test is to highlight the shear locking phenomenon, which particularly affects linear solid finite elements, when employed to model thin structures. This test has been proposed by Kögl and Bucalem [59], and it is used to assess the effectiveness of the various treatments adopted to prevent such locking phenomena. For this purpose, a square plate of side $1000 \mathrm{~mm}$ and thickness $a_{0}$ is considered. The plate is clamped at one side and is bent by applying a line force at the opposite edge as shown in Fig. 5. This steel plate is covered by a PZT piezoelectric sensor, which has a width of $200 \mathrm{~mm}$ and a thickness $a_{1}$. The material properties are reported in Table 3. For different configurations of thicknesses, we determine the tip displacement $U_{z}^{\mathrm{C}}$ at point $\mathrm{C}$ (see Fig. 5). The reference solution is obtained with a refined mesh using the ABAQUS quadratic element C3D20E.

The results in terms of normalized tip displacements at point $\mathrm{C}$ are reported in Table 4 for different plate thicknesses and geometric ratios. It appears that with the SHB8PSE solid-shell element, the relative error is comprised between $1.1 \%\left(a_{0}=10 \mathrm{~mm} ; a_{1} / a_{0}=0.01\right)$ and $4.5 \%$ $\left(a_{0}=1 \mathrm{~mm} ; a_{1} / a_{0}=0.001\right)$. By contrast, using the same mesh of $20 \times 4 \times 1$ hexahedral elements, the C3D8E element exhibits very poor results due to its high sensitivity to locking effects. Moreover, the C3D8E shows high sensitivity to the element aspect ratio, as it provides results that are 1000 times underestimated, for $a_{0}=1 \mathrm{~mm}$, to almost 2 times overestimated, for $a_{0}=100 \mathrm{~mm}$. Therefore, to achieve better results with the C3D8E element, much more refined meshes would be required, thereby significantly increasing the CPU time. 
This benchmark test also demonstrates the effectiveness of the assumed-strain projection technique applied to the piezoelectric solid-shell element SHB8PSE to prevent various locking phenomena.

\subsubsection{Extension and shear piezoelectric actuation mechanisms}

In this third benchmark test, we consider two configurations of cantilever sandwich beams, as illustrated in Fig. 6, which are actuatable in extension (a) and in shear (b), respectively. These tests represent excellent benchmark problems for their selectivity, and have become popular, as commonly studied in a number of literature works, including those of Zhang and Sun $[60,61]$ and Benjeddou et al. [62].

The materials are polarized in the $z$-direction for the extension mechanism, and in the $x$ direction for the shear mechanism. In order to bend the beam, voltages are applied to the upper and lower surfaces of the piezoelectric layers, inducing electric bending forces. Thus, for the shear actuation mechanism, a voltage of $\Delta \phi=20 \mathrm{~V}$ is applied to the piezoelectric core, while for the extension actuation mechanism, voltages of $\Delta \phi= \pm 10 \mathrm{~V}$ are applied to the surface of the actuators. The corresponding material properties are all reported in Table 5. The numerical results obtained with the proposed SHB8PSE and SHB20E piezoelectric solidshell elements are compared, on the one hand, with those taken from the literature [60-62] and, on the other hand, with those given by state-of-the-art ABAQUS elements that have equivalent geometry and kinematics, namely the C3D8E and C3D20E piezoelectric solid elements.

Two cases with regard to the piezoelectric layer arrangement are considered. The first case corresponds to the situation when the piezoelectric layers cover the entire length of the beam; while in the second case, the position of the actuator varies in the 10-90 $\mathrm{mm}$ range. We start by analyzing the first case, and the corresponding simulation results are reported in Fig. 7. Note that, in this first case and for the shear actuation mechanism, there is no rigid foam and, instead, the piezoelectric actuator covers the entire core layer. It appears from Fig. 7 that the results obtained with the proposed SHB8PSE and SHB20E elements are in excellent agreement with those given by ABAQUS elements, while systematically requiring a fewer number of elements to achieve convergence. However, the literature results, which are obtained by $2 \mathrm{D}$ modeling, seem to overestimate the transverse deflection in the case when the beam is actuated by piezoelectric expansion (see Fig. 7-a).

In the second case, where the actuator position varies in the $10-90 \mathrm{~mm}$ range, the deflection at the free edge is investigated for each position of the piezoelectric patches. Here again, the simulation results are compared with those taken from the literature (Benjeddou et al. [62] and Piefort [63], where only shear mechanism results are available) as well as with those given by the ABAQUS linear and quadratic piezoelectric solid elements C3D8E and C3D20E. From Fig. 8, it is observed that the results obtained with the SHB8PSE and SHB20E elements are in good agreement with those of the literature as well as with those yielded by ABAQUS elements, for both actuation mechanisms. However, it is worth noting that the results obtained with the proposed solid-shell elements converge faster than those of existing 
conventional elements (i.e., relatively fewer elements are required with the SHB8PSE and SHB20E formulations to achieve convergence, as shown in Fig. 8).

\subsubsection{Square plate with piezoelectric patch models}

One important advantage taken from the piezoelectric behavior is in the application to the shape control of structures. In order to show the interest of solid-shell finite elements in this type of modeling, we consider a square aluminum plate of $200 \times 200 \mathrm{~mm}^{2}$ with a thickness of 8 $\mathrm{mm}$. This plate is covered on both sides with four pairs of localized PZT-5H patches in various configurations, as shown in Fig. 9. Each patch has dimensions of $40 \times 40 \mathrm{~mm}^{2}$ with a thickness of $1 \mathrm{~mm}$. The objective of this test is to investigate the optimal location of piezoelectric patches for shape control. With regard to loading conditions, the plate is subjected to a uniformly distributed load of $100 \mathrm{~N} . \mathrm{m}^{-2}$ over its entire surface. A constant voltage is then supplied incrementally to the piezoceramic actuators, which are polarized in opposite directions, until the plate is flattened. Fig. 10 shows the centerline deflection of the composite plate along the $x$-direction under different input voltages. The results provided by the solid-shell elements SHB8PSE and SHB20E are compared with those given by the ABAQUS solid elements C3D8E and C3D20E. On the whole, it appears that fewer overall degrees of freedom are required for the proposed solid-shell elements to achieve convergence, as compared to ABAQUS elements. It is also noteworthy that despite the high number of overall degrees of freedom required by the $\mathrm{C} 3 \mathrm{D} 8 \mathrm{E}$ element, it provides less accurate results than the SHB8PSE, especially in configuration (c), where the error margin reaches $16 \%$.

In addition, the analysis of the plots in Fig. 10 shows that the (a) and (d) configurations are more effective in terms of shape control (plate flatness recovery). Note however that configuration (a) requires up to $-20 \mathrm{~V}$ by pair of patches to recover the initial shape of the plate, whereas only $2 \mathrm{~V}$ are sufficient for configuration $(\mathrm{d})$.

\subsubsection{Shallow cylindrical sandwich blade}

In order to assess the capabilities of the proposed solid-shell elements in geometric nonlinear analysis, a cantilever shallow cylindrical sandwich shell with $300 \mathrm{~mm}$ for both of its straight and curved edges, as depicted in Fig. 11, is considered. A similar model has been proposed by Kioua and Mirza [64], but no comparison with available finite element technologies was attempted. Here again, the host shell is made in aluminum and has a thickness of $2.50 \mathrm{~mm}$. This shell is entirely covered on both sides with a thin PZT-5H layer of $0.25 \mathrm{~mm}$ thickness polarized across the thickness. A voltage of $50 \mathrm{~V}$ is applied to each piezoelectric layer (the internal faces are connected to ground, while $50 \mathrm{~V}$ is applied to the external faces) to induce bending actuation. Three values for the $R / b$ ratio are considered $(R / b=\{1,10, \infty\})$. The considered layup configuration for the laminated shell causes high stiffness coupling and, consequently, also generates a twisting deformation. The deflections along paths A, B and C, as shown in Fig. 11, are investigated. 
In Fig. 12, the results provided by the solid-shell finite elements SHB8PSE and SHB20E are compared to those given by the ABAQUS solid elements C3D8E and C3D20E. Once again, the good behavior of the SHB8PSE and SHB20E elements is clearly observed, which highlights the benefit of using the proposed solid-shell elements in this kind of analysis.

With this preliminary set of static tests performed, focus is placed in the following sections on free vibration modeling of sandwich structures that contain piezoelectric layers, in order to evaluate the performance of the proposed solid-shell formulations.

\subsection{Vibration test problems}

In the case of free vibration analysis, the system of discretized equations (4) becomes

$$
\left(\left[\begin{array}{ll}
\mathbf{K}^{u u} & \mathbf{K}^{u \phi} \\
\mathbf{K}^{\phi u} & \mathbf{K}^{\phi \phi}
\end{array}\right]-\omega^{2}\left[\begin{array}{cc}
\mathbf{M}^{u u} & \mathbf{0} \\
\mathbf{0} & \mathbf{0}
\end{array}\right]\right)\left\{\begin{array}{l}
\boldsymbol{U} \\
\boldsymbol{\phi}
\end{array}\right\}=\left\{\begin{array}{l}
\mathbf{0} \\
\mathbf{0}
\end{array}\right\}
$$

In the following sections, a set of free vibration tests both in open-circuit and short-circuit configurations will be carried out on beam, plate and shell structures.

\subsubsection{Beam benchmark tests}

We consider here two sandwich beam models. These benchmark tests are similar to those previously presented in static analysis with shear and extension actuation mechanisms; however, they have different geometric parameters, as shown in Fig. 13. The elastic layers are made of aluminum, while the piezoelectric layers are made in PZT-5H material. The modal analysis is performed using both the short-circuit (SC) and open-circuit (OC) configurations. In Tables 6 and 7, the first five free vibration frequencies are provided, revealing that the results obtained with the SHB8PSE and SHB20E elements are in good agreement with those given by the ABAQUS quadratic solid element C3D20E. It should be emphasized, however, that less overall degrees of freedom are required with the proposed solid-shell elements to achieve accurate results, as compared to their ABAQUS solid element counterparts. It is also worth noting that, despite the higher number of overall degrees of freedom required by the C3D8E element, its results still fall far from the reference solutions, especially for high frequencies.

\subsubsection{Sandwich plate}

In this second example of this category of benchmark problems, we investigate the free vibration response of a simply supported sandwich plate. The plate consists of two piezoelectric faces, in PZT-5H material polarized along the thickness, covering a core made of aluminum with a varying thickness. The piezoelectric faces have a thickness of $1 \mathrm{~mm}$, while the other geometric dimensions are shown in Fig. 14. Different thicknesses for the aluminum core are considered, according to a geometric ratio $r$, in order to analyze the sensitivity to thickness reduction of the results given by the proposed solid-shell elements. 
The first five free vibration frequencies are investigated in both short-circuit and open-circuit configurations and are reported in Table 8.

According to Table 8, the results obtained with the solid-shell finite elements SHB8PSE and SHB20E are in good agreement with those of the reference ABAQUS element C3D20E. It should be noted, however, that fewer degrees of freedom are required for the proposed solid-shell elements, as compared to their counterparts with the same kinematics. Note also that, despite the finer mesh required by the C3D8E element, its results fall most of the time far from the reference solutions.

\subsubsection{Cantilever plate with piezo-patches}

This benchmark test has been proposed by Cinefra et al. [65] and concerns the free vibration analysis of a cantilever aluminum plate with four pairs of piezoelectric patches. More specifically, four patches are bonded on the top surface, with the four others symmetrically bonded on the bottom surface (see Fig. 15). The material and geometric properties of the structure are reported in Table 9. The first ten free vibration frequencies are investigated in both short-circuit and open-circuit configurations and are reported in Table 10. The results obtained with the proposed solid-shell elements are compared to those yielded by ABAQUS elements and also to those given in Cinefra et al. [65]. The latter are based on a model derived from the CUF with finite element discretization employing the 9-node MITC element (Mixed Interpolation of Tensorial Components). The analysis in Table 10 shows that the results obtained with the proposed solid-shell elements are in good agreement with those of the reference element C3D20E as well as with those given by the CUF-MITC model [65]. It also clearly appears that the linear ABAQUS solid element C3D8E, despite the high number of overall degrees of freedom, provides very poor results due to its high sensitivity to locking effects.

In the following sections, free vibration analysis of shell structures provided with piezoelectric materials will be conducted. The aim is to assess the performance and reliability of the proposed solid-shell elements in the modeling of sandwich structures involving geometric nonlinearities. In this latter context, two benchmark tests will be analyzed.

\subsubsection{Curved cantilever sandwich blade}

In this test problem, a curved cantilever sandwich blade is considered. A similar test has been investigated by Zouari et al. [66], based on the experimentations conducted by Olson and Lindberg [67], but only involved purely elastic behavior. Here, similar to the previously studied test, the core is made of aluminum, while the faces are made in PZT-5H material, as shown in Fig. 16. Several core thicknesses are also investigated both in short-circuit and open-circuit configurations. From the results reported in Table 11, it appears that, once again, the conventional solid finite elements C3D8E and C3D20E require finer meshes to provide accurate results, as compared to the proposed solid-shell formulations. One may also notice that with 4 times more elements for the C3D8E, as compared to the SHB8PSE element, it still 
exhibits a margin of error that exceeds $20 \%$, for the fifth frequency and $\mathrm{r}=1$, while the relative error with the SHB8PSE is of about $4 \%$ only.

\subsubsection{Hemispherical sandwich shell with a hole}

The last benchmark test in this category is concerned with a doubly curved sandwich shell structure. This consists of a hemispherical shell, with an $18^{\circ}$ hole and a mean radius of 200 $\mathrm{mm}$, as depicted in Fig. 17. The host structure is made of aluminum material and has a thickness of $1.50 \mathrm{~mm}$. This hemispherical shell is entirely covered on both sides with a thin PZT-5H layer, which is polarized across its thickness of $0.25 \mathrm{~mm}$. The shell structure is clamped over the entire holed face. The current analysis consists in investigating the first five modes in both short-circuit and open-circuit configurations, which are illustrated in Fig. 18. The results in terms of the corresponding natural frequencies (first five natural frequencies) are summarized in Table 12.

As previously done, the results obtained with the proposed solid-shell formulations are compared in Table 12 with their ABAQUS counterparts, which are based on the same geometry and kinematics. From Table 12, it appears that, while using coarser meshes, the results provided by the developed solid-shell elements are in good agreement with the reference solution given by the C3D20E ABAQUS quadratic piezoelectric element. Again, the worst results are by far those provided by the C3D8E ABAQUS solid element, which is attributable to its high sensitivity to locking effects.

\section{Summary and conclusions}

In the current contribution, two new hexahedral piezoelectric solid-shell finite elements have been developed. These finite element technologies consist of an eight-node hexahedron, denoted as SHB8PSE, and a twenty-node hexahedron, designated as SHB20E. These formulations are based on purely three-dimensional kinematics and, accordingly, the resulting finite elements have at each of their nodes three translational degrees of freedom and one electric degree of freedom. To provide these elements with some desirable shell features, and to alleviate locking effects, an in-plane reduced-integration scheme is adopted, with a userdefined number of integration points along the thickness direction. The constitutive law is also expressed in a local physical coordinate system, which is attached to the element midplane, in order to enhance immunity with regard to thickness locking.

Particular attention has been paid to the polarization of piezoelectric patches, which plays a very important role in the mechanism of deformation. Both static and free vibration benchmark tests have been successfully conducted on structures ranging from simple beams and plates to more complex sandwich shells. The simulation results obtained with the newly devised solid-shell elements have been compared with reference solutions taken from the literature and also with state-of-the-art finite elements available in ABAQUS. Among the latter, the quadratic hexahedral piezoelectric solid element C3D20E is often taken as reference. In all of the benchmark tests investigated, the solid-shell elements SHB8PSE and 
SHB20E have shown better performance, as compared to their ABAQUS counterparts, the C3D8E and C3D20E, respectively, while systematically necessitating a fewer number of elements for similar accuracy. Also, although in some situations of severe nonlinearities the SHB8PSE element may exhibit some over-stiffness due to locking, its accuracy and convergence rate remain overall much better than those of the C3D8E element. In future work, it would be interesting to further improve the performance of the SHB8PSE element, by using for instance other advanced enhanced assumed strain methods. It would be also interesting to extend this study to the modeling of vibration control of multilayer structures with piezoelectric materials in complete layers or patches.

Appendix A. Derivation of the discrete gradient operators

For the SHB8PSE solid-shell element, the combination of Eqs. (5) and (6), along with the expression of the shape functions for linear eight-node elements, allows expanding the displacement field in the following form:

$$
\left\{\begin{array}{l}
u_{i}=a_{0 i}+a_{1 i} x_{1}+a_{2 i} x_{2}+a_{3 i} x_{3}+c_{1 i} h_{1}+c_{2 i} h_{2}+c_{3 i} h_{3}+c_{4 i} h_{4} \\
h_{1}=\eta \zeta, h_{2}=\xi \zeta, h_{3}=\xi \eta, h_{4}=\xi \eta \zeta
\end{array}\right.
$$

By evaluating equation (A.1) at the nodes of the element, one can obtain a set of eightequation systems defined by

$$
\begin{aligned}
\boldsymbol{d}_{i}= & a_{0 i} \boldsymbol{s}+a_{1 i} \boldsymbol{x}_{1}+a_{2 i} \boldsymbol{x}_{2}+a_{3 i} \boldsymbol{x}_{3}+c_{1 i} \boldsymbol{h}_{1}+c_{2 i} \boldsymbol{h}_{2}+c_{3 i} \boldsymbol{h}_{3}+c_{4 i} \boldsymbol{h}_{4} \\
& i=1,2,3
\end{aligned}
$$

where $\boldsymbol{d}_{i}$ and $\boldsymbol{x}_{i}$ denote the nodal displacements and nodal coordinates, respectively, while vectors $\boldsymbol{s}$ and $\boldsymbol{h}_{\alpha}$ are defined by

$$
\left\{\begin{array}{l}
\boldsymbol{s}^{T}=(1,1,1,1,1,1,1,1) \\
\boldsymbol{h}_{1}^{T}=(1,1,-1,-1,-1,-1,1,1) \\
\boldsymbol{h}_{2}^{T}=(1,-1,-1,1,-1,1,1,-1) \\
\boldsymbol{h}_{3}^{T}=(1,-1,1,-1,1,-1,1,-1) \\
\boldsymbol{h}_{4}^{T}=(-1,1,-1,1,1,-1,1,-1)
\end{array}\right.
$$

To determine the yet unknown constants $a_{j i}$ and $c_{\alpha i}$, the derivatives of the shape functions evaluated at the origin of the reference frame are introduced 


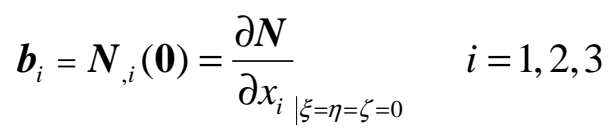

Finally, using a set of preliminarily established orthogonality relations, one obtains

$$
\begin{aligned}
& a_{j i}=\boldsymbol{b}_{j}^{T} \cdot \boldsymbol{d}_{i} \quad ; \quad c_{\alpha i}=\boldsymbol{\gamma}_{\alpha}^{T} \cdot \boldsymbol{d}_{i} \\
& \text { where } \gamma_{\alpha}=\frac{1}{8}\left[\boldsymbol{h}_{\alpha}-\sum_{j=1}^{3}\left(\boldsymbol{h}_{\alpha}^{T} \cdot \boldsymbol{x}_{j}\right) \boldsymbol{b}_{j}\right], \quad \alpha=1, \ldots, 4
\end{aligned}
$$

In the case of the SHB20E solid-shell element, combining Eqs. (5) and (6), in conjunction with the explicit form of the shape functions for quadratic twenty-node elements, leads to the following expansion for the displacement field:

$$
\left\{\begin{aligned}
u_{i}= & a_{0 i}+a_{1 i} x_{1}+a_{2 i} x_{2}+a_{3 i} x_{3}+c_{1 i} h_{1}+c_{2 i} h_{2}+c_{3 i} h_{3}+c_{4 i} h_{4}+c_{5 i} h_{5} \\
& +c_{6 i} h_{6}+c_{7 i} h_{7}+c_{8 i} h_{8}+c_{9 i} h_{9}+c_{10 i} h_{10}+c_{11 i} h_{11}+c_{12 i} h_{12}+c_{13 i} h_{13} \\
& +c_{14 i} h_{14}+c_{15 i} h_{15}+c_{16 i} h_{16} \\
h_{1}= & \xi \zeta, h_{2}=\eta \zeta, h_{3}=\xi \eta, h_{4}=\xi^{2}, h_{5}=\eta^{2}, h_{6}=\zeta^{2}, h_{7}=\xi \eta \zeta, \\
h_{8}= & \xi^{2} \eta, h_{9}=\xi^{2} \zeta, h_{10}=\eta^{2} \xi, h_{11}=\eta^{2} \zeta, h_{12}=\zeta^{2} \xi, h_{13}=\zeta^{2} \eta, \\
h_{14}= & \xi^{2} \eta \zeta, h_{15}=\xi \eta^{2} \zeta, h_{16}=\xi \eta \zeta^{2}
\end{aligned}\right.
$$

Similarly, the evaluation of equation (A.6) at the nodes of the element leads to three systems of 20 equations defined by

$$
\begin{aligned}
\boldsymbol{d}_{i}= & a_{0 i} \boldsymbol{s}+a_{1 i} \boldsymbol{x}_{1}+a_{2 i} \boldsymbol{x}_{2}+a_{3 i} \boldsymbol{x}_{3}+c_{1 i} \boldsymbol{h}_{1}+c_{2 i} \boldsymbol{h}_{2}+c_{3 i} \boldsymbol{h}_{3}+\cdots+c_{16 i} \boldsymbol{h}_{16} \\
& i=1,2,3
\end{aligned}
$$

where $\boldsymbol{d}_{i}$ and $\boldsymbol{x}_{i}$ represent the nodal displacements and nodal coordinates, respectively, while vectors $\boldsymbol{s}$ and $\boldsymbol{h}_{\alpha}$ are defined by 


$$
\left\{\begin{array}{l}
\boldsymbol{s}^{T}=(1,1,1,1,1,1,1,1,1,1,1,1,1,1,1,1,1,1,1,1) \\
\boldsymbol{h}_{1}^{T}=(1,-1,-1,1,-1,1,1,-1,0,-1,0,1,0,0,0,0,0,1,0,-1) \\
\boldsymbol{h}_{2}^{T}=(1,1,-1,-1,-1,-1,1,1,1,0,-1,0,0,0,0,0,-1,0,1,0) \\
\boldsymbol{h}_{3}^{T}=(1,-1,1,-1,1,-1,1,-1,0,0,0,0,1,-1,1,-1,0,0,0,0) \\
\boldsymbol{h}_{4}^{T}=(1,1,1,1,1,1,1,1,0,1,0,1,1,1,1,1,0,1,0,1) \\
\boldsymbol{h}_{5}^{T}=(1,1,1,1,1,1,1,1,1,0,1,0,1,1,1,1,1,0,1,0) \\
\boldsymbol{h}_{6}^{T}=(1,1,1,1,1,1,1,1,1,1,1,1,0,0,0,0,1,1,1,1) \\
\boldsymbol{h}_{7}^{T}=(-1,1,-1,1,1,-1,1,-1,0,0,0,0,0,0,0,0,0,0,0,0) \\
\boldsymbol{h}_{8}^{T}=(-1,-1,1,1,-1,-1,1,1,0,0,0,0,-1,-1,1,1,0,0,0,0) \\
\boldsymbol{h}_{9}^{T}=(-1,-1,-1,-1,1,1,1,1,0,-1,0,-1,0,0,0,0,0,1,0,1) \\
\boldsymbol{h}_{10}^{T}=(-1,1,1,-1,-1,1,1,-1,0,0,0,0,-1,1,1,-1,0,0,0,0) \\
\boldsymbol{h}_{11}^{T}=(-1,-1,-1,-1,1,1,1,1,-1,0,-1,0,0,0,0,0,1,0,1,0) \\
\boldsymbol{h}_{12}^{T}=(-1,1,1,-1,-1,1,1,-1,0,1,0,-1,0,0,0,0,0,1,0,-1) \\
\boldsymbol{h}_{13}^{T}=(-1,-1,1,1,-1,-1,1,1,-1,0,1,0,0,0,0,0,-1,0,1,0) \\
\boldsymbol{h}_{14}^{T}=(1,1,-1,-1,-1,-1,1,1,0,0,0,0,0,0,0,0,0,0,0,0) \\
\boldsymbol{h}_{15}^{T}=(1,-1,-1,1,-1,1,1,-1,0,0,0,0,0,0,0,0,0,0,0,0) \\
\boldsymbol{h}_{16}^{T}=(1,-1,1,-1,1,-1,1,-1,0,0,0,0,0,0,0,0,0,0,0,0)
\end{array}\right.
$$

In a similar way, using some preliminarily established orthogonality conditions, it can be shown that the yet unknown constants $a_{j i}$ and $c_{\alpha i}$ are given by the same Eq. (A.5). However, the previous expressions of vectors $\gamma_{\alpha}$ must be replaced here by

$$
\begin{aligned}
\boldsymbol{\gamma}_{\alpha} & =n_{\alpha 1}\left(\boldsymbol{h}_{1}-\left(\boldsymbol{h}_{1}^{T} \cdot \boldsymbol{x}_{j}\right) \boldsymbol{b}_{j}\right)+n_{\alpha 2}\left(\boldsymbol{h}_{2}-\left(\boldsymbol{h}_{2}^{T} \cdot \boldsymbol{x}_{j}\right) \boldsymbol{b}_{j}\right)+n_{\alpha 3}\left(\boldsymbol{h}_{3}-\left(\boldsymbol{h}_{3}^{T} \cdot \boldsymbol{x}_{j}\right) \boldsymbol{b}_{j}\right) \\
& +n_{\alpha 4}\left[\left(\boldsymbol{h}_{4}-\frac{4}{5} \boldsymbol{s}\right)-\left(\left(\boldsymbol{h}_{4}^{T}-\frac{4}{5} \boldsymbol{s}^{T}\right) \cdot \boldsymbol{x}_{j}\right) \boldsymbol{b}_{j}\right]+n_{\alpha 5}\left[\left(\boldsymbol{h}_{5}-\frac{4}{5} \boldsymbol{s}\right)-\left(\left(\boldsymbol{h}_{5}^{T}-\frac{4}{5} \boldsymbol{s}^{T}\right) \cdot \boldsymbol{x}_{j}\right) \boldsymbol{b}_{j}\right] \\
& +n_{\alpha 6}\left[\left(\boldsymbol{h}_{6}-\frac{4}{5} \boldsymbol{s}\right)-\left(\left(\boldsymbol{h}_{6}^{T}-\frac{4}{5} \boldsymbol{s}^{T}\right) \cdot \boldsymbol{x}_{j}\right) \boldsymbol{b}_{j}\right]+n_{\alpha 7}\left(\boldsymbol{h}_{7}-\left(\boldsymbol{h}_{7}^{T} \cdot \boldsymbol{x}_{j}\right) \cdot \boldsymbol{b}_{j}\right) \\
& +n_{\alpha 8}\left(\boldsymbol{h}_{8}-\left(\boldsymbol{h}_{8}^{T} \cdot \boldsymbol{x}_{j}\right) \cdot \boldsymbol{b}_{j}\right)+n_{\alpha 9}\left(\boldsymbol{h}_{9}-\left(\boldsymbol{h}_{9}^{T} \cdot \boldsymbol{x}_{j}\right) \cdot \boldsymbol{b}_{j}\right)+n_{\alpha 10}\left(\boldsymbol{h}_{10}-\left(\boldsymbol{h}_{10}^{T} \cdot \boldsymbol{x}_{j}\right) \cdot \boldsymbol{b}_{j}\right) \\
& +n_{\alpha 11}\left(\boldsymbol{h}_{11}-\left(\boldsymbol{h}_{11}^{T} \cdot \boldsymbol{x}_{j}\right) \cdot \boldsymbol{b}_{j}\right)+n_{\alpha 12}\left(\boldsymbol{h}_{12}-\left(\boldsymbol{h}_{12}^{T} \cdot \boldsymbol{x}_{j}\right) \cdot \boldsymbol{b}_{j}\right)+n_{\alpha 13}\left(\boldsymbol{h}_{13}-\left(\boldsymbol{h}_{13}^{T} \cdot \boldsymbol{x}_{j}\right) \cdot \boldsymbol{b}_{j}\right) \\
& +n_{\alpha 14}\left(\boldsymbol{h}_{14}-\left(\boldsymbol{h}_{14}^{T} \cdot \boldsymbol{x}_{j}\right) \cdot \boldsymbol{b}_{j}\right)+n_{\alpha 15}\left(\boldsymbol{h}_{15}-\left(\boldsymbol{h}_{15}^{T} \cdot \boldsymbol{x}_{j}\right) \cdot \boldsymbol{b}_{j}\right)+n_{\alpha 16}\left(\boldsymbol{h}_{16}-\left(\boldsymbol{h}_{16}^{T} \cdot \boldsymbol{x}_{j}\right) \cdot \boldsymbol{b}_{j}\right)
\end{aligned}
$$

where the constant components $n_{\alpha \beta}$ are given by 


$$
\left[n_{\alpha \beta}\right]=\left[\begin{array}{cccccccccccccccc}
\frac{1}{4} & 0 & 0 & 0 & 0 & 0 & 0 & 0 & 0 & 0 & 0 & 0 & 0 & 0 & -\frac{1}{4} & 0 \\
0 & \frac{1}{4} & 0 & 0 & 0 & 0 & 0 & 0 & 0 & 0 & 0 & 0 & 0 & -\frac{1}{4} & 0 & 0 \\
0 & 0 & \frac{1}{4} & 0 & 0 & 0 & 0 & 0 & 0 & 0 & 0 & 0 & 0 & 0 & 0 & -\frac{1}{4} \\
0 & 0 & 0 & \frac{3}{8} & \frac{1}{8} & \frac{1}{8} & 0 & 0 & 0 & 0 & 0 & 0 & 0 & 0 & 0 & 0 \\
0 & 0 & 0 & \frac{1}{8} & \frac{3}{8} & \frac{1}{8} & 0 & 0 & 0 & 0 & 0 & 0 & 0 & 0 & 0 & 0 \\
0 & 0 & 0 & \frac{1}{8} & \frac{1}{8} & \frac{3}{8} & 0 & 0 & 0 & 0 & 0 & 0 & 0 & 0 & 0 & 0 \\
0 & 0 & 0 & 0 & 0 & 0 & \frac{1}{8} & 0 & 0 & 0 & 0 & 0 & 0 & 0 & 0 & 0 \\
0 & 0 & 0 & 0 & 0 & 0 & 0 & \frac{3}{20} & 0 & 0 & 0 & 0 & -\frac{1}{10} & 0 & 0 & 0 \\
0 & 0 & 0 & 0 & 0 & 0 & 0 & 0 & 0 & \frac{3}{20} & 0 & -\frac{1}{10} & 0 & 0 & 0 & 0 \\
0 & 0 & 0 & 0 & 0 & 0 & 0 & 0 & -\frac{1}{10} & 0 & \frac{3}{20} & 0 & 0 & 0 & 0 & 0 \\
0 & 0 & 0 & 0 & 0 & 0 & 0 & 0 & 0 & -\frac{1}{10} & 0 & \frac{3}{20} & 0 & 0 & 0 & 0 \\
0 & 0 & 0 & 0 & 0 & 0 & 0 & -\frac{1}{10} & 0 & 0 & 0 & 0 & \frac{3}{20} & 0 & 0 & 0 \\
0 & -\frac{1}{4} & 0 & 0 & 0 & 0 & 0 & 0 & 0 & 0 & 0 & 0 & 0 & \frac{1}{8} & 0 & 0 \\
-\frac{1}{4} & 0 & 0 & 0 & 0 & 0 & 0 & 0 & 0 & 0 & 0 & 0 & 0 & 0 & \frac{1}{8} & 0 \\
0 & 0 & -\frac{1}{4} & 0 & 0 & 0 & 0 & 0 & 0 & 0 & 0 & 0 & 0 & 0 & 0 & \frac{1}{8}
\end{array}\right]
$$

Appendix B. Computation of the stabilization matrices for the SHB8PSE

As mentioned before, the stabilization matrices for the SHB8PSE element are computed in a co-rotational coordinate frame before being transformed to the orthotropy frame of the piezoelectric material. In this orthogonal co-rotational coordinate frame, which has been previously defined by a rotation matrix $\boldsymbol{R}$ given by Eqs. (13)-(15), several terms of the stabilization matrices are shown to simplify. Indeed, because this system of co-rotational coordinates is chosen to be aligned with the reference frame, the relationships between the two coordinate systems can be approximated by

$$
\left\{\begin{array}{l}
\frac{\partial \tilde{x}_{i}}{\partial \xi_{i}}=\frac{1}{\partial \xi_{i} / \partial \tilde{x}_{i}}=\frac{1}{8} \boldsymbol{\Lambda}_{i}^{T} \cdot \tilde{\boldsymbol{x}}_{i} \\
\frac{\partial \tilde{x}_{i}}{\partial \xi_{j}}=\frac{\partial \xi_{j}}{\partial \tilde{x}_{i}}=0 \quad ; \quad i \neq j
\end{array}\right.
$$


where vector $\tilde{\boldsymbol{x}}_{i}$ refers to the nodal coordinates expressed in the co-rotational frame, and repeated indices here do not indicate a summation rule. Then, using Eq. (B.1), the following simplifications are demonstrated (see [53] for more details):

$$
h_{i, i}=\frac{\partial h_{i}}{\partial \tilde{x}_{i}}=0, \quad h_{j, i}=\frac{8 \xi_{k}}{\boldsymbol{\Lambda}_{i}^{T} \cdot \tilde{\boldsymbol{x}}_{i}}, \quad h_{4, i}=\frac{8 \xi_{j} \xi_{k}}{\boldsymbol{\Lambda}_{i}^{T} \cdot \tilde{\boldsymbol{x}}_{i}}, \quad \tilde{J}=\frac{\boldsymbol{\Lambda}_{1}^{T} \cdot \tilde{\boldsymbol{x}}_{1}}{8} \frac{\boldsymbol{\Lambda}_{2}{ }^{T} \cdot \tilde{\boldsymbol{x}}_{2}}{8} \frac{\boldsymbol{\Lambda}_{3}{ }^{T} \cdot \tilde{\boldsymbol{x}}_{3}}{8}
$$

where $\tilde{J}$ denotes the determinant of the Jacobian matrix. Note again that the repeated indices in Eq. (B.2) do not indicate a summation rule. In addition, the indices $i, j$ and $k$ are pairwise distinct and take values 1, 2 and 3, with all of the possible permutations. Finally, Eq. (B.2) leads to the following expressions:

$$
\begin{aligned}
& \int_{V_{e}} h_{i, j} d v=0 \\
& \left\{\begin{array}{l}
H_{i i}=\int_{V_{e}}\left(h_{j, i}\right)^{2} d v=\int_{V_{e}}\left(h_{k, i}\right)^{2} d v=3 \int_{V_{e}}\left(h_{4, i}\right)^{2} d v=\frac{1}{3} \frac{\left(\boldsymbol{\Lambda}_{j}^{T} \cdot \tilde{\boldsymbol{x}}_{j}\right)\left(\boldsymbol{\Lambda}_{k}{ }^{T} \cdot \tilde{\boldsymbol{x}}_{k}\right)}{\left(\boldsymbol{\Lambda}_{i}{ }^{T} \cdot \tilde{\boldsymbol{x}}_{i}\right)} \\
H_{i j}=\int_{V_{e}} h_{i, j} h_{j, i} d v=\frac{1}{3}\left(\boldsymbol{\Lambda}_{k}^{T} \cdot \tilde{\boldsymbol{x}}_{k}\right)
\end{array}\right.
\end{aligned}
$$

By replacing the expressions (B.3) in Eq. (12), providing the stabilization stiffness matrix, one obtains

$$
\mathbf{K}_{\text {Stab }}^{u u}=\left[\begin{array}{lll}
\mathbf{k}_{11} & \mathbf{k}_{12} & \mathbf{k}_{13} \\
\mathbf{k}_{21} & \mathbf{k}_{22} & \mathbf{k}_{23} \\
\mathbf{k}_{31} & \mathbf{k}_{32} & \mathbf{k}_{33}
\end{array}\right]
$$

where the $8 \times 8$ matrices $\mathbf{k}_{i j}$ are given by

$$
\left\{\begin{array}{l}
\mathbf{k}_{11}=(\bar{\lambda}+2 \mu) H_{11}\left[\hat{\gamma}_{3} \hat{\gamma}_{3}^{T}+\frac{1}{3} \hat{\gamma}_{4} \hat{\gamma}_{4}^{T}\right] \\
\mathbf{k}_{22}=(\bar{\lambda}+2 \mu) H_{22}\left[\hat{\gamma}_{3} \hat{\gamma}_{3}^{T}+\frac{1}{3} \hat{\gamma}_{4} \hat{\gamma}_{4}^{T}\right] \\
\mathbf{k}_{33}=(\bar{\lambda}+2 \mu) H_{11} \frac{1}{3} \hat{\gamma}_{4} \hat{\gamma}_{4}^{T} \\
\mathbf{k}_{i j}=\mathbf{0}, \quad i \neq j
\end{array}\right.
$$

where $\bar{\lambda}=E v /\left(1-v^{2}\right)$ and $\mu=E / 2(1+v)$, with $E$ being the Young modulus and $v$ the Poisson ratio. 
Similarly, the stabilization matrices $\mathbf{K}_{\text {Stab }}^{u \phi}$ and $\mathbf{K}_{\text {Stab }}^{\phi \phi}$, related to the piezoelectric and dielectric matrices, are obtained as

$$
\begin{aligned}
& \mathbf{K}_{\text {Stab }}^{u \phi}=\left[\begin{array}{l}
\mathbf{k}_{11}^{u \phi} \\
\mathbf{k}_{21}^{u \phi} \\
\mathbf{k}_{31}^{u \phi}
\end{array}\right] ; \quad\left\{\begin{array}{l}
\mathbf{k}_{11}^{u \phi}=e_{31} H_{11}\left[\hat{\gamma}_{3} \hat{\gamma}_{3}^{T}+\frac{1}{3} \hat{\gamma}_{4} \hat{\gamma}_{4}^{T}\right] \\
\mathbf{k}_{21}^{u \phi}=e_{32} H_{22}\left[\hat{\gamma}_{3} \hat{\gamma}_{3}^{T}+\frac{1}{3} \hat{\gamma}_{4} \hat{\gamma}_{4}^{T}\right] \\
\mathbf{k}_{31}^{u \phi}=\mathbf{0}
\end{array}\right. \\
& \mathbf{K}_{\text {Stab }}^{\phi \phi}=-\left[\mathbf{k}_{11}^{\phi \phi}\right] ; \quad \mathbf{k}_{11}^{\phi \phi}=\kappa_{31} H_{11}\left[\hat{\gamma}_{3} \hat{\gamma}_{3}^{T}+\frac{1}{3} \hat{\gamma}_{4} \hat{\gamma}_{4}^{T}\right]
\end{aligned}
$$

Appendix C. Transformation of tensors from local (material) frame to global frame

The piezoelectric properties of the materials are specified in a local orthotropy frame inherent to the material. For the computation of the different matrices involved (e.g., stiffness, piezoelectric and dielectric permittivity matrices), it is necessary to evaluate these properties in a global fixed coordinate system. For this purpose, a rotation matrix $\boldsymbol{R}$ is used for the transformation of vector and matrix components from the global frame to the local physical frame. Hence, the components $\mathrm{C}_{i j k l}, \mathrm{e}_{i j k}$ and $\kappa_{i j}$ of the tensors $\mathbf{C}, \mathbf{e}$ and $\boldsymbol{\kappa}$, expressed in the global coordinate system, are related to their components $\hat{\mathrm{C}}_{m n o p}, \hat{\mathrm{e}}_{m n o}$ and $\hat{\mathrm{\kappa}}_{m n}$ in the local material frame by the following relationships:

$$
\left\{\begin{array}{l}
\mathrm{C}_{i j k l}=\boldsymbol{R}_{i m} \boldsymbol{R}_{j n} \boldsymbol{R}_{k o} \boldsymbol{R}_{l p} \hat{\mathrm{C}}_{m n o p} \\
\mathrm{e}_{i j k}=\boldsymbol{R}_{i m} \boldsymbol{R}_{j n} \boldsymbol{R}_{k o} \hat{\mathrm{e}}_{m n o} \\
\boldsymbol{\kappa}_{i j}=\boldsymbol{R}_{i m} \boldsymbol{R}_{j n} \hat{\kappa}_{m n}
\end{array}\right.
$$

In matrix form, Eq. (C.1) can be rewritten as

$$
\left\{\begin{array}{l}
\mathbf{C}=\boldsymbol{T} \cdot \hat{\mathbf{C}} \cdot \boldsymbol{T}^{T} \\
\mathbf{e}=\boldsymbol{R} \cdot \hat{\mathbf{e}} \cdot \boldsymbol{T}^{T} \\
\mathbf{K}=\boldsymbol{R} \cdot \hat{\mathbf{K}} \cdot \boldsymbol{R}^{T}
\end{array}\right.
$$

where 


$$
\boldsymbol{R}=\left[\begin{array}{lll}
\boldsymbol{R}_{11} & \boldsymbol{R}_{12} & \boldsymbol{R}_{13} \\
\boldsymbol{R}_{21} & \boldsymbol{R}_{22} & \boldsymbol{R}_{23} \\
\boldsymbol{R}_{31} & \boldsymbol{R}_{32} & \boldsymbol{R}_{33}
\end{array}\right]
$$

and

$$
\boldsymbol{T}=\left[\begin{array}{cccccc}
\boldsymbol{R}_{11}^{2} & \boldsymbol{R}_{12}^{2} & \boldsymbol{R}_{13}^{2} & \boldsymbol{R}_{12} \boldsymbol{R}_{13} & \boldsymbol{R}_{11} \boldsymbol{R}_{13} & \boldsymbol{R}_{11} \boldsymbol{R}_{12} \\
\boldsymbol{R}_{21}^{2} & \boldsymbol{R}_{22}^{2} & \boldsymbol{R}_{33}^{2} & \boldsymbol{R}_{22} \boldsymbol{R}_{23} & \boldsymbol{R}_{21} \boldsymbol{R}_{23} & \boldsymbol{R}_{22} \boldsymbol{R}_{21} \\
\boldsymbol{R}_{31}^{2} & \boldsymbol{R}_{32}^{2} & \boldsymbol{R}_{33}^{2} & \boldsymbol{R}_{33} \boldsymbol{R}_{32} & \boldsymbol{R}_{33} \boldsymbol{R}_{31} & \boldsymbol{R}_{31} \boldsymbol{R}_{32} \\
\boldsymbol{R}_{21} \boldsymbol{R}_{31} & \boldsymbol{R}_{22} \boldsymbol{R}_{32} & \boldsymbol{R}_{23} \boldsymbol{R}_{33} & \boldsymbol{R}_{22} \boldsymbol{R}_{33}+\boldsymbol{R}_{23} \boldsymbol{R}_{32} & \boldsymbol{R}_{21} \boldsymbol{R}_{33}+\boldsymbol{R}_{23} \boldsymbol{R}_{31} & \boldsymbol{R}_{22} \boldsymbol{R}_{31}+\boldsymbol{R}_{21} \boldsymbol{R}_{32} \\
\boldsymbol{R}_{11} \boldsymbol{R}_{31} & \boldsymbol{R}_{12} \boldsymbol{R}_{32} & \boldsymbol{R}_{13} \boldsymbol{R}_{33} & \boldsymbol{R}_{12} \boldsymbol{R}_{33}+\boldsymbol{R}_{13} \boldsymbol{R}_{32} & \boldsymbol{R}_{13} \boldsymbol{R}_{31}+\boldsymbol{R}_{11} \boldsymbol{R}_{33} & \boldsymbol{R}_{11} \boldsymbol{R}_{32}+\boldsymbol{R}_{12} \boldsymbol{R}_{31} \\
\boldsymbol{R}_{11} \boldsymbol{R}_{21} & \boldsymbol{R}_{12} \boldsymbol{R}_{22} & \boldsymbol{R}_{13} \boldsymbol{R}_{23} & \boldsymbol{R}_{12} \boldsymbol{R}_{23}+\boldsymbol{R}_{13} \boldsymbol{R}_{22} & \boldsymbol{R}_{13} \boldsymbol{R}_{21}+\boldsymbol{R}_{11} \boldsymbol{R}_{23} & \boldsymbol{R}_{11} \boldsymbol{R}_{22}+\boldsymbol{R}_{12} \boldsymbol{R}_{21}
\end{array}\right]
$$

\section{References}

[1] K. Takayoshi, F. Takafumi, H. Takayoshi, A. Takeo, M. Nobuyoshi, A. Satoru, and T. Kohtaro, "Active vibration control of frame structures with smart structures using piezoelectric actuators (Vibration control by control of bending moments of columns)," Smart Materials and Structures, vol. 6, pp. 448-456, 1997.

[2] X. G. Tan and L. Vu-Quoc, "Optimal solid-shell element for large-deformable composite structures with piezoelectric layers and active vibration control," International Journal for Numerical Methods in Engineering, vol. 64, pp. 1981-2013, 2005.

[3] S. Belouettar, L. Azrar, E. M. Daya, V. Laptev, and M. Potier-Ferry, "Active control of nonlinear vibration of sandwich piezoelectric beams: A simplified approach," Computers \& Structures, vol. 86, pp. 386-397, 2008.

[4] C. Chee, L. Tong, and G. P. Steven, "Piezoelectric actuator orientation optimization for static shape control of composite plates," Composite Structures, vol. 55, pp. 169$184,2002$.

[5] Q. Nguyen and L. Tong, "Shape control of smart composite plate with non-rectangular piezoelectric actuators," Composite Structures, vol. 66, pp. 207-214, 2004.

[6] Q. Nguyen and L. Tong, "Voltage and evolutionary piezoelectric actuator design optimisation for static shape control of smart plate structures," Materials \& Design, vol. 28, pp. 387-399, 2007.

[7] X. Zhang, J. Lu, and Y. Shen, "Active noise control of flexible linkage mechanism with piezoelectric actuators," Computers \& Structures, vol. 81, pp. 2045-2051, 2003. 
[8] S. M. Hasheminejad and M. Rajabi, "Scattering and active acoustic control from a submerged piezoelectric-coupled orthotropic hollow cylinder," Journal of Sound and Vibration, vol. 318, pp. 50-73, 2008.

[9] A. S. Wixom, M. J. Anderson, D. F. Bahr, and D. J. Morris, "A new acoustic transducer with a pressure-deformed piezoelectric diaphragm," Sensors and Actuators A: Physical, vol. 179, pp. 204-210, 2012.

[10] H. F. Olson, "Electronic control of noise, vibration and reverberation," Journal of the Acoustical Society of America, vol. 28, pp. 972-976, 1956.

[11] G. Park, H. Cudney, and D. Inman, "Impedance-based health monitoring of civil structural components," Journal of Infrastructure Systems, vol. 6, pp. 153-160, 2000.

[12] W. H. Duan, Q. Wang, and S. T. Quek, "Applications of piezoelectric materials in structural health monitoring and repair: Selected Research Examples," Materials, vol. 3, pp. 5169-5194, 2010.

[13] Y. K. An, M. K. Kim, and H. Sohn, "Piezoelectric transducers for assessing and monitoring civil infrastructures," Sensor Technologies for Civil Infrastructures, vol. 1, pp. 86-120, 2014.

[14] H. Allik and T. J. R. Hughes, "Finite element method for piezoelectric vibration," International Journal for Numerical Methods in Engineering, vol. 2, pp. 151-157, 1970.

[15] D. A. Saravanos and P. R. Heyliger, "Coupled layerwise analysis of composite beams with embedded piezoelectric sensors and actuators," Journal of Intelligent Material Systems and Structures, vol. 6, pp. 350-363, 1995.

[16] A. Benjeddou, "Advances in piezoelectric finite element modeling of adaptive structural elements: a survey," Computers \& Structures, vol. 76, pp. 347-363, 2000.

[17] T. Rödig, A. Schönecker, and G. Gerlach, "A survey on piezoelectric ceramics for generator applications," Journal of the American Ceramic Society, vol. 93, pp. 901912, 2010.

[18] J. Peng and X. Chen, "A survey of modeling and control of piezoelectric actuators," Modern Mechanical Engineering, vol. 3, pp. 1-20, 2013.

[19] J.-H. Han and I. Lee, "Analysis of composite plates with piezoelectric actuators for vibration control using layerwise displacement theory," Composites Part B: Engineering, vol. 29, pp. 621-632, 1998.

[20] W.-S. Hwang and H. C. Park, "Finite element modeling of piezoelectric sensors and actuators," AIAA Journal, vol. 31, pp. 930-937, 1993.

[21] D. H. Robbins and J. N. Reddy, "Analysis of piezoelectrically actuated beams using a layer-wise displacement theory," Computers \& Structures, vol. 41, pp. 265-279, 1991. 
[22] G. R. Liu, X. Q. Peng, K. Y. Lam, and J. Tani, "Vibration control simulation of laminated composite plates with integrated piezoelectrics," Journal of Sound and Vibration, vol. 220, pp. 827-846, 1999.

[23] J. M. Simões Moita, I. F. P. Correia, C. M. Mota Soares, and C. A. Mota Soares, "Active control of adaptive laminated structures with bonded piezoelectric sensors and actuators," Computers \& Structures, vol. 82, pp. 1349-1358, 2004.

[24] R. D. Mindlin, "Forced thickness $\square$ shear and flexural vibrations of piezoelectric crystal plates," Journal of Applied Physics, vol. 23, pp. 83-88, 1952.

[25] J. M. S. Moita, C. M. M. Soares, and C. A. M. Soares, "Active control of forced vibrations in adaptive structures using a higher order model," Composite Structures, vol. 71, pp. 349-355, 2005.

[26] T. S. Plagianakos and E. G. Papadopoulos, "Higher-order 2-D/3-D layerwise mechanics and finite elements for composite and sandwich composite plates with piezoelectric layers," Aerospace Science and Technology, vol. 40, pp. 150-163, 2015.

[27] A. Mannini and P. Gaudenzi, "Multi-layer higher-order finite elements for the analysis of free-edge stresses in piezoelectric actuated laminates," Composite Structures, vol. 63, pp. 263-270, 2004.

[28] S. M. Shiyekar and T. Kant, "Higher order shear deformation effects on analysis of laminates with piezoelectric fibre reinforced composite actuators," Composite Structures, vol. 93, pp. 3252-3261, 2011.

[29] S. Kapuria, P. C. Dumir, A. Ahmed, and N. Alam, "Finite element model of efficient zig-zag theory for static analysis of hybrid piezoelectric beams," Computational Mechanics, vol. 34, pp. 475-483, 2004.

[30] S. Kapuria, A. Ahmed, and P. C. Dumir, "An efficient coupled zigzag theory for dynamic analysis of piezoelectric composite and sandwich beams with damping," Journal of Sound and Vibration, vol. 279, pp. 345-371, 2005.

[31] S. Kapuria and N. Alam, "Efficient layerwise finite element model for dynamic analysis of laminated piezoelectric beams," Computer Methods in Applied Mechanics and Engineering, vol. 195, pp. 2742-2760, 2006.

[32] S. Kapuria and S. D. Kulkarni, "Efficient finite element with physical and electric nodes for transient analysis of smart piezoelectric sandwich plates," Acta Mechanica, vol. 214, pp. 123-131, 2010.

[33] H. Boudaoud, E. M. Daya, S. Belouettar, L. Duigou, and M. Potier-Ferry, "Damping analysis of beams submitted to passive and active control," Engineering Structures, vol. 31, pp. 322-331, 2009.

[34] L. Azrar, S. Belouettar, and J. Wauer, "Nonlinear vibration analysis of actively loaded sandwich piezoelectric beams with geometric imperfections," Computers \& Structures, vol. 86, pp. 2182-2191, 2008. 
[35] D. Ballhause, M. D’Ottavio, B. Kröplin, and E. Carrera, "A unified formulation to assess multilayered theories for piezoelectric plates," Computers \& Structures, vol. 83, pp. 1217-1235, 2005.

[36] A. Robaldo, E. Carrera, and A. Benjeddou, "A unified formulation for finite element analysis of piezoelectric adaptive plates," Computers \& Structures, vol. 84, pp. 1494$1505,2006$.

[37] E. Carrera and M. Boscolo, "Classical and mixed finite elements for static and dynamic analysis of piezoelectric plates," International Journal for Numerical Methods in Engineering, vol. 70, pp. 1135-1181, 2007.

[38] E. Carrera, M. Boscolo, and A. Robaldo, "Hierarchic multilayered plate elements for coupled multifield problems of piezoelectric adaptive structures: formulation and numerical assessment," Archives of Computational Methods in Engineering, vol. 14, pp. 383-430, 2007.

[39] E. Carrera and S. Brischetto, "Piezoelectric shell theories with a priori continuous transverse electromechanical variables," Journal of Mechanics of Materials and Structures, vol. 2, pp. 377-398, 2007.

[40] E. Carrera and P. Nali, "Mixed piezoelectric plate elements with direct evaluation of transverse electric displacement," International Journal for Numerical Methods in Engineering, vol. 80, pp. 403-424, 2009.

[41] E. Carrera and P. Nali, "Multilayered plate elements for the analysis of multifield problems," Finite Elements in Analysis and Design, vol. 46, pp. 732-742, 2010.

[42] K. Y. Sze and L. Q. Yao, "A hybrid stress ANS solid-shell element and its generalization for smart structure modelling. Part I-solid-shell element formulation," International Journal for Numerical Methods in Engineering, vol. 48, pp. 545-564, 2000 .

[43] K. Y. Sze, L. Q. Yao, and S. Yi, "A hybrid stress ANS solid-shell element and its generalization for smart structure modelling. Part II-smart structure modelling," International Journal for Numerical Methods in Engineering, vol. 48, pp. 565-582, 2000 .

[44] S. Klinkel and W. Wagner, "A geometrically non-linear piezoelectric solid shell element based on a mixed multi-field variational formulation," International Journal for Numerical Methods in Engineering, vol. 65, pp. 349-382, 2006.

[45] S. Klinkel and W. Wagner, "A piezoelectric solid shell element based on a mixed variational formulation for geometrically linear and nonlinear applications," Computers \& Structures, vol. 86, pp. 38-46, 2008.

[46] S. Zheng, X. Wang, and W. Chen, "The formulation of a refined hybrid enhanced assumed strain solid shell element and its application to model smart structures containing distributed piezoelectric sensors/actuators," Smart Materials and Structures, vol. 13, pp. 43-50, 2004. 
[47] G. M. Kulikov and S. V. Plotnikova, "Geometrically exact four-node piezoelectric solid-shell element," Mechanics of Advanced Materials and Structures, vol. 15, pp. 199-207, 2008.

[48] G. M. Kulikov and S. V. Plotnikova, "The use of 9-parameter shell theory for development of exact geometry 12-node quadrilateral piezoelectric laminated solidshell elements," Mechanics of Advanced Materials and Structures, vol. 22, pp. 490$502,2014$.

[49] T. Nestorović, D. Marinković, G. Chandrashekar, Z. Marinković, and M. Trajkov, "Implementation of a User Defined piezoelectric shell element for analysis of active structures," Finite Elements in Analysis and Design, vol. 52, pp. 11-12, 2012.

[50] T. Nestorović, S. Shabadi, D. Marinković, and M. Trajkov, "Modeling of piezoelectric smart structures by implementation of a User Defined shell finite element," Mechanical Engineering, vol. 11, pp. 1-12, 2013.

[51] T. Nestorović, D. Marinković, S. Shabadi, and M. Trajkov, "User defined finite element for modeling and analysis of active piezoelectric shell structures," Meccanica, vol. 49, pp. 1763-1774, 2014.

[52] F. Abed-Meraim and A. Combescure, "SHB8PS - A new adaptative, assumed-strain continuum mechanics shell element for impact analysis," Computers \& Structures, vol. 80, pp. 791-803, 2002.

[53] F. Abed-Meraim and A. Combescure, "An improved assumed strain solid-shell element formulation with physical stabilization for geometric non-linear applications and elastic-plastic stability analysis," International Journal for Numerical Methods in Engineering, vol. 80, pp. 1640-1686, 2009.

[54] M. Killpack and F. Abed-Meraim, "Limit-point buckling analyses using solid, shell and solid-shell elements," Journal of Mechanical Science and Technology, vol. 25, pp. 1105-1117, 2011.

[55] F. Abed-Meraim, V. D. Trinh, and A. Combescure, "New quadratic solid-shell elements and their evaluation on linear benchmark problems," Computing, vol. 95, pp. 373-394, 2013.

[56] D. P. Flanagan and T. Belytschko, "A uniform strain hexahedron and quadrilateral with orthogonal hourglass control," International Journal for Numerical Methods in Engineering, vol. 17, pp. 679-706, 1981.

[57] T. Belytschko and L. P. Bindeman, "Assumed strain stabilization of the eight node hexahedral element," Computer Methods in Applied Mechanics and Engineering, vol. 105, pp. 225-260, 1993.

[58] H. S. Tzou and R. Ye, "Analysis of piezoelastic structures with laminated piezoelectric triangle shell elements," AIAA Journal, vol. 34, pp. 110-115, 1996.

[59] M. Kögl and M. L. Bucalem, "Analysis of smart laminates using piezoelectric MITC plate and shell elements," Computers \& Structures, vol. 83, pp. 1153-1163, 2005. 
[60] X. D. Zhang and C. T. Sun, "Formulation of an adaptive sandwich beam," Smart Materials and Structures, vol. 5, pp. 814-823, 1996.

[61] C. T. Sun and X. D. Zhang, "Use of thickness-shear mode in adaptive sandwich structures," Smart Materials and Structures, vol. 4, pp. 202-206, 1995.

[62] A. Benjeddou, M. Trindade, and R. Ohayon, "A unified beam finite element model for extension and shear piezoelectric actuation mechanisms," Journal of Intelligent Material Systems and Structures, vol. 8, pp. 1012-1025, 1997.

[63] V. Piefort, "Finite element modeling of piezoelectric active structures," $\mathrm{PhD}$ Thesis, University of Brussels, 2001.

[64] H. Kioua and S. Mirza, "Piezoelectric induced bending and twisting of laminated composite shallow shells," Smart Materials and Structures, vol. 9, pp. 476-484, 2000.

[65] M. Cinefra, S. Valvano, and E. Carrera, "A layer-wise MITC9 finite element for the free-vibration analysis of plates with piezo-patches," International Journal of Smart and Nano Materials, vol. 6, pp. 85-104, 2015.

[66] W. Zouari, M. Assarar, K. Meftah, and R. Ayad, "Free vibration analysis of homogeneous piezoelectric structures using specific hexahedral elements with rotational DOFs," Acta Mechanica, vol. 226, pp. 1737-1756, 2015.

[67] M. D. Olson and G. M. Lindberg, "Dynamic analysis of shallow shells with a doublycurved triangular finite element," Journal of Sound and Vibration, vol. 19, pp. 299318, 1971. 


\section{Tables}

Table 1. Explicit forms for the matrices and vectors resulting from the electromechanical coupling.

\begin{tabular}{ll}
\hline$\left[\mathbf{M}^{u u}\right]=\int_{V}\left[\boldsymbol{N}^{u}\right]^{T} \rho\left[\boldsymbol{N}^{u}\right] d v$ & Mass matrix \\
{$\left[\mathbf{K}^{u u}\right]=\int_{V}\left[\mathbf{B}^{u}\right]^{T}[\mathbf{C}]\left[\mathbf{B}^{u}\right] d v$} & Stiffness matrix \\
{$\left[\mathbf{K}^{\phi \phi}\right]=-\int_{V}\left[\mathbf{B}^{\phi}\right]^{T}[\mathbf{K}]\left[\mathbf{B}^{\phi}\right] d v$} & Dielectric matrix \\
{$\left[\mathbf{K}^{u \phi}\right]=\int_{V}\left[\mathbf{B}^{u}\right]^{T}[\mathbf{e}]^{T}\left[\mathbf{B}^{\phi}\right] d v ;\left[\mathbf{K}^{\phi u}\right]=\left[\mathbf{K}^{u \phi}\right]^{T}$} & Piezoelectric coupling matrix \\
$\{\boldsymbol{F}\}=\int_{V}\left[\boldsymbol{N}^{u}\right]^{T}\left\{\boldsymbol{f}_{v}\right\} d v+\int_{S}\left[\boldsymbol{N}^{u}\right]^{T}\left\{\boldsymbol{f}_{s}\right\} d s+\boldsymbol{f}_{p}$ & Force vector \\
$\{\boldsymbol{Q}\}=-\int_{V}\left[\boldsymbol{N}^{\phi}\right]^{T}\left\{\boldsymbol{q}_{v}\right\} d v-\int_{S}\left[\boldsymbol{N}^{\phi}\right]^{T}\left\{\boldsymbol{q}_{s}\right\} d s-\boldsymbol{q}_{p}$ & Electrical charge vector \\
\hline
\end{tabular}

Table 2. Tip displacements at $x=L$.

\begin{tabular}{ccccccccc}
\hline$E$ & & $\mathrm{e}_{31}$ & $\mathrm{e}_{32}$ & $\kappa_{31}$ & \multicolumn{4}{c}{$U_{z}(\mu \mathrm{m})$} \\
\cline { 6 - 9 }$(\mathrm{GPa})$ & $v$ & $\left({\left.\mathrm{C} / \mathrm{m}^{2}\right)}\right.$ & $\left({\left.\mathrm{C} / \mathrm{m}^{2}\right)}(\mu \mathrm{F} / \mathrm{m})\right.$ & $\begin{array}{c}\mathrm{C} 3 \mathrm{D} 8 \mathrm{E} \\
(100 \times 4 \times 2)\end{array}$ & $\begin{array}{c}\text { SHB8PSE } \\
(10 \times 1 \times 2)\end{array}$ & $\begin{array}{c}\text { C3D20E } \\
(10 \times 1 \times 2)\end{array}$ & $\begin{array}{c}\mathrm{SHB} 20 \mathrm{E} \\
(5 \times 1 \times 2)\end{array}$ \\
\hline 2 & 0 & -0.046 & -0.046 & 0.1062 & 0.258 & 0.345 & 0.344 & 0.345 \\
\hline
\end{tabular}

Table 3. Material properties for the steel plate with a piezoelectric sensor layer; permittivity

$$
\kappa_{0}=8.854 \times 10^{-8} \mathrm{~F} / \mathrm{m} \text {, with } \kappa_{i i}=\kappa_{0} \cdot \kappa_{i i}^{\mathrm{rel}} \text {. }
$$

\begin{tabular}{ll}
\hline & $\mathrm{C}_{11}=\mathrm{C}_{22}=107.60 \mathrm{GPa} ; \mathrm{C}_{33}=100.40 \mathrm{GPa}$ \\
& $\mathrm{C}_{12}=63.12 \mathrm{GPa} ; \mathrm{C}_{13}=\mathrm{C}_{23}=84.1 \mathrm{GPa}$ \\
& $\mathrm{C}_{44}=\mathrm{C}_{55}=19.62 \mathrm{GPa} ; \mathrm{C}_{66}=22.24 \mathrm{GPa}$ \\
& $\mathrm{e}_{15}=\mathrm{e}_{24}=12.0 \mathrm{C} / \mathrm{m}^{2}$ \\
& $\mathrm{e}_{31}=\mathrm{e}_{32}=-9.6 \mathrm{C} / \mathrm{m}^{2} ; \mathrm{e}_{33}=15.1 \mathrm{C} / \mathrm{m}^{2}$ \\
& $\kappa_{11}^{\text {rel }}=\kappa_{22}^{\text {rel }}=1936 ; \kappa_{33}^{\text {rel }}=2109$ \\
\hline Steel & $\mathrm{E}=210 \mathrm{GPa} ; v=0.3$ \\
\hline
\end{tabular}


Table 4. Locking effects that may affect a thin piezoelectric sensor (thickness $a_{1}$, discretized with piezoelectric elements) attached to a steel plate (thickness $a_{0}$, discretized with solid or solid-shell elements): normalized displacements $U_{z}^{\mathrm{C}} / U_{z}^{\mathrm{C} \text {,ref }}$.

\begin{tabular}{clccc}
\hline$a_{0}[\mathrm{~mm}]$ & \multicolumn{1}{c}{$a_{1} / a_{0}$} & 0.001 & 0.01 & 0.1 \\
\hline \multirow{3}{*}{1} & C3D8 $(20 \times 20 \times 1)+\mathrm{C} 3 \mathrm{D} 8 \mathrm{E}(20 \times 4 \times 1)$ & 0.001 & 0.001 & 0.001 \\
& SHB8PS $(20 \times 20 \times 1)+$ SHB8PSE $(20 \times 4 \times 1)$ & 1.045 & 1.038 & 0.983 \\
& C3D20 $(10 \times 10 \times 1)+$ C3D20E $(10 \times 2 \times 1)$ & 0.958 & 0.957 & 0.956 \\
& SHB20 $(10 \times 10 \times 1)+$ SHB20E $(10 \times 2 \times 1)$ & 0.996 & 0.996 & 0.995 \\
\hline \multirow{4}{*}{10} & C3D8 $(20 \times 20 \times 1)+$ C3D8E $(20 \times 4 \times 1)$ & 0.108 & 0.108 & 0.107 \\
& SHB8PS $(20 \times 20 \times 1)+$ SHB8PSE $(20 \times 4 \times 1)$ & 1.034 & 1.011 & 0.974 \\
& C3D20 $(10 \times 10 \times 1)+$ C3D20E $(10 \times 2 \times 1)$ & 0.968 & 0.966 & 0.944 \\
& SHB20 $(10 \times 10 \times 1)+$ SHB20E $(10 \times 2 \times 1)$ & 0.998 & 0.997 & 0.997 \\
\hline \multirow{4}{*}{100} & C3D8 $(20 \times 20 \times 1)+$ C3D8E $(20 \times 4 \times 1)$ & 1.865 & 1.858 & 1.776 \\
& SHB8PS $(20 \times 20 \times 1)+$ SHB8PSE $(20 \times 4 \times 1)$ & 1.028 & 1.023 & 0.975 \\
& C3D20 $(10 \times 10 \times 1)+$ C3D20E $(10 \times 2 \times 1)$ & 0.981 & 0.979 & 0.957 \\
& SHB20 $(10 \times 10 \times 1)+$ SHB20E $(10 \times 2 \times 1)$ & 0.999 & 0.999 & 0.999 \\
\hline
\end{tabular}

Table 5. Material properties used in the extension and shear mechanism models.

\begin{tabular}{ll}
\hline & $\rho=7730 \mathrm{Kg} \cdot \mathrm{m}^{-3}$ \\
& $\mathrm{C}_{11}=\mathrm{C}_{22}=\mathrm{C}_{33}=126 \mathrm{GPa}$ \\
& $\mathrm{C}_{12}=79.5 \mathrm{GPa} ; \mathrm{C}_{13}=\mathrm{C}_{23}=84.1 \mathrm{GPa}$ \\
& $\mathrm{C}_{44}=\mathrm{C}_{55}=\mathrm{C}_{66}=23 \mathrm{GPa}$ \\
& $\mathrm{e}_{15}=\mathrm{e}_{24}=17 \mathrm{C} / \mathrm{m}^{2}$ \\
& $\mathrm{e}_{31}=\mathrm{e}_{32}=-6.5 \mathrm{C} / \mathrm{m}^{2} ; \mathrm{e}_{33}=23.3 \mathrm{C} / \mathrm{m}^{2}$ \\
& $\kappa_{11}=\kappa_{22}=1.503 \times 10^{-8} \mathrm{~F} / \mathrm{m} ; \kappa_{33}=1.3 \times 10^{-8} \mathrm{~F} / \mathrm{m}$ \\
& \\
\hline \multirow{2}{*}{ Foam } & $\rho=32 \mathrm{Kg} \cdot \mathrm{m}^{-3}$ \\
& $\mathrm{E}=35.3 \mathrm{MPa} ; v=0.38$ \\
\hline \multirow{2}{*}{ Aluminum $\quad \rho=2690 \mathrm{Kg} \cdot \mathrm{m}^{-3}$} \\
$\mathrm{E}=70.3 \mathrm{GPa} ; v=0.345$
\end{tabular}


Table 6. First five natural frequencies for the extension actuated sandwich beam.

\begin{tabular}{ccccc}
\hline & $\begin{array}{c}\text { C3D8E } \\
(40 \times 4 \times 4)\end{array}$ & $\begin{array}{c}\text { SHB8PSE } \\
(20 \times 2 \times 3)\end{array}$ & $\begin{array}{c}\text { C3D20E } \\
(20 \times 2 \times 3)\end{array}$ & $\begin{array}{c}\text { SHB20E } \\
(10 \times 1 \times 3)\end{array}$ \\
\hline Short circuit & & & & \\
\hline 1 & 764.42 & 754.72 & 751.58 & 749.35 \\
2 & 1271.0 & 1275.8 & 1271.2 & 1268.46 \\
3 & 4705.9 & 4655.5 & 4630.3 & 4616.0 \\
4 & 7610.9 & 7658.6 & 7615.7 & 7600.8 \\
5 & 8926.7 & 9223.6 & 9293.6 & 9253.3 \\
\hline Open circuit & & & & \\
\hline 1 & 805.66 & 789.10 & 787.17 & 785.76 \\
2 & 1294.9 & 1301.6 & 1297.4 & 1294.8 \\
3 & 4951.4 & 4861.4 & 4842.5 & 4833.3 \\
4 & 7744.8 & 7805.3 & 7763.3 & 7750.6 \\
5 & 8930.7 & 9261.9 & 9297.4 & 9296.0 \\
\hline
\end{tabular}

Table 7. First five natural frequencies for the shear actuated sandwich beam.

\begin{tabular}{|c|c|c|c|c|}
\hline & $\begin{array}{c}\text { C3D8E } \\
(40 \times 4 \times 3)\end{array}$ & $\begin{array}{l}\text { SHB8PSE } \\
(20 \times 2 \times 3)\end{array}$ & $\begin{array}{l}\text { C3D20E } \\
(20 \times 2 \times 3)\end{array}$ & $\begin{array}{l}\text { SHB20E } \\
(10 \times 1 \times 3)\end{array}$ \\
\hline \multicolumn{5}{|c|}{ Short circuit $\equiv$ Open circuit } \\
\hline 1 & 791.36 & 789.10 & 780.87 & 781.61 \\
\hline 2 & 1271.7 & 1276.8 & 1271.8 & 1269.2 \\
\hline 3 & 4875.2 & 4873.4 & 4813.2 & 4817.5 \\
\hline 4 & 7618.6 & 7668.6 & 7623.8 & 7610.8 \\
\hline 5 & 9912.4 & 10501 & 10475 & 10434 \\
\hline
\end{tabular}


Table 8. First five natural frequencies for the rectangular sandwich plate.

\begin{tabular}{|c|c|c|c|c|}
\hline & $\begin{array}{c}\text { C3D8E } \\
(48 \times 40 \times 3)\end{array}$ & $\begin{array}{l}\text { SHB8PSE } \\
(24 \times 20 \times 3)\end{array}$ & $\begin{array}{c}\text { C3D20E } \\
(24 \times 20 \times 3)\end{array}$ & $\begin{array}{c}\text { SHB20E } \\
(12 \times 10 \times 3)\end{array}$ \\
\hline \multicolumn{5}{|c|}{ Short circuit } \\
\hline \multirow{5}{*}{$\mathrm{r}=1$} & 223.95 & 185.98 & 183.23 & 181.09 \\
\hline & 425.59 & 341.49 & 333.22 & 330.27 \\
\hline & 530.56 & 405.12 & 392.41 & 390.66 \\
\hline & 728.22 & 605.46 & 584.85 & 586.70 \\
\hline & 824.87 & 640.87 & 606.99 & 605.60 \\
\hline \multirow{5}{*}{$r=5$} & 507.81 & 512.84 & 514.54 & 506.96 \\
\hline & 912.90 & 939.91 & 930.65 & 919.43 \\
\hline & 1087.2 & 1115.0 & 1095.1 & 1088.2 \\
\hline & 1577.4 & 1660.3 & 1628.7 & 1620.1 \\
\hline & 1687.0 & 1766.7 & 1697.8 & 1695.0 \\
\hline \multirow{5}{*}{$\mathrm{r}=20$} & 1458.5 & 1704.7 & 1712.3 & 1707.4 \\
\hline & 2451.2 & 3003.2 & 3004.0 & 2875.3 \\
\hline & 2789.5 & 3406.6 & 3441.1 & 3385.4 \\
\hline & 4143.4 & 5159.3 & 5125.0 & 5146.7 \\
\hline & 4372.4 & 5603.9 & 5426.7 & 5522.3 \\
\hline \multicolumn{5}{|c|}{ Open circuit } \\
\hline \multirow{5}{*}{$\mathrm{r}=1$} & 244.31 & 210.04 & 207.35 & 205.20 \\
\hline & 457.24 & 382.95 & 374.25 & 372.21 \\
\hline & 566.96 & 455.22 & 441.48 & 439.92 \\
\hline & 786.27 & 680.54 & 658.43 & 660.02 \\
\hline & 884.27 & 723.84 & 686.53 & 685.81 \\
\hline \multirow{5}{*}{$r=5$} & 552.26 & 557.74 & 558.40 & 551.45 \\
\hline & 994.08 & 1021.7 & 1010.5 & 1003.5 \\
\hline & 1184.0 & 1214.5 & 1191.5 & 1185.1 \\
\hline & 1723.6 & 1806.6 & 1771.0 & 1764.1 \\
\hline & 1840.7 & 1927.8 & 1851.0 & 1848.5 \\
\hline \multirow{5}{*}{$\mathrm{r}=20$} & 1533.0 & 1764.6 & 1771.2 & 1758.9 \\
\hline & 2579.5 & 3126.3 & 3099.4 & 3000.0 \\
\hline & 2939.2 & 3618.3 & 3551.9 & 3495.7 \\
\hline & 4363.5 & 5335.9 & 5282.7 & 5307.2 \\
\hline & 4606.1 & 5786.0 & 5596.6 & 5661.0 \\
\hline
\end{tabular}


Table 9. Material and geometric data for the cantilever plate with piezoelectric patches.

\begin{tabular}{ll}
\hline & $\mathrm{a}_{0}=0.075 \mathrm{~m} ; \mathrm{b}_{0}=0.025 \mathrm{~m} ; \mathrm{h}_{0}=0.63 \times 10^{-3} \mathrm{~m}$ \\
& $\rho=7600 \mathrm{Kg} \cdot \mathrm{m}^{-3}$ \\
& $\mathrm{C}_{11}=\mathrm{C}_{22}=\mathrm{C}_{33}=90.37 \mathrm{GPa}$ \\
& $\mathrm{C}_{12}=\mathrm{C}_{13}=\mathrm{C}_{23}=35.14 \mathrm{GPa}$ \\
& $\mathrm{C}_{44}=\mathrm{C}_{55}=\mathrm{C}_{66}=24.8 \mathrm{GPa}$ \\
& $\mathrm{e}_{15}=\mathrm{e}_{24}=16.62 \mathrm{C} / \mathrm{m}^{2}$ \\
& $\mathrm{e}_{31}=\mathrm{e}_{32}=-14.47 \mathrm{C} / \mathrm{m}^{2} ; \mathrm{e}_{33}=18.34 \mathrm{C} / \mathrm{m}^{2}$ \\
& $\kappa_{11}=\kappa_{22}=15.3 \times 10^{-9} \mathrm{~F} / \mathrm{m} ; \kappa_{33}=15.0 \times 10^{-9} \mathrm{~F} / \mathrm{m}$ \\
\hline & $\mathrm{a}=0.3 \mathrm{~m} ; \mathrm{b}=0.2 \mathrm{~m} ; \mathrm{h}=0.8 \times 10^{-3} \mathrm{~m}$ \\
& $\rho=2700 \mathrm{Kg} \cdot \mathrm{m}^{-3}$ \\
& $\mathrm{E}=70 \mathrm{GPa} ; v=0.32$
\end{tabular}


Table 10. First ten free frequencies for the cantilever plate with piezo-patches.

\begin{tabular}{lccccc}
\hline & C3D8 & SHB8PS & C3D20 & SHB20 & \\
& $(80 \times 32 \times 1)$ & $(40 \times 16 \times 1)$ & $(40 \times 16 \times 1)$ & $(20 \times 8 \times 1)$ & CUF - MITC \\
& + & + & + & + & {$[65]$} \\
& C3D8E & SHB8PSE & C3D20E & SHB20E & $(20 \times 8)$ \\
& $8 \times(20 \times 4 \times 1)$ & $8 \times(10 \times 2 \times 1)$ & $8 \times(10 \times 2 \times 1)$ & $8 \times(5 \times 1 \times 1)$ & \\
\hline Short circuit & & & & & \\
\hline 1 & 19.395 & 7.5679 & 7.6138 & 7.7271 & 7.6059 \\
2 & 37.296 & 25.426 & 25.904 & 25.762 & 25.346 \\
3 & 126.11 & 45.696 & 45.975 & 46.299 & 45.787 \\
4 & 164.34 & 90.648 & 92.159 & 91.601 & 90.446 \\
5 & 347.01 & 124.63 & 124.96 & 124.30 & 123.76 \\
6 & 369.33 & 148.86 & 148.79 & 151.52 & 148.93 \\
7 & 496.42 & 184.67 & 185.48 & 185.49 & 184.69 \\
8 & 579.36 & 237.29 & 241.54 & 239.96 & 235.50 \\
9 & 714.57 & 332.27 & 329.74 & 327.58 & 328.22 \\
10 & 737.00 & 332.50 & 333.28 & 335.31 & 332.49 \\
\hline Open circuit & & & & & \\
\hline 1 & 19.540 & 7.6114 & 7.6547 & 7.7681 & 7.6074 \\
2 & 37.420 & 25.478 & 25.959 & 25.829 & 25.351 \\
3 & 126.43 & 45.762 & 46.043 & 46.366 & 45.790 \\
4 & 164.69 & 90.892 & 92.425 & 91.926 & 90.454 \\
5 & 351.48 & 124.77 & 125.09 & 124.42 & 123.76 \\
6 & 374.30 & 150.69 & 150.61 & 153.48 & 149.00 \\
7 & 496.52 & 186.69 & 187.52 & 189.81 & 184.88 \\
8 & 579.63 & 237.55 & 241.84 & 240.34 & 235.48 \\
9 & 718.10 & 333.81 & 334.51 & 326.42 & 327.03 \\
10 & 746.49 & 339.34 & 336.93 & 343.08 & 332.31 \\
\hline
\end{tabular}


Table 11. First five natural frequencies for the curved cantilever sandwich blade.

\begin{tabular}{|c|c|c|c|c|}
\hline & $\begin{array}{c}\text { C3D8E } \\
(48 \times 40 \times 3)\end{array}$ & $\begin{array}{l}\text { SHB8PSE } \\
(24 \times 20 \times 3)\end{array}$ & $\begin{array}{c}\text { C3D20E } \\
(24 \times 20 \times 3)\end{array}$ & $\begin{array}{c}\text { SHB20E } \\
(12 \times 10 \times 3)\end{array}$ \\
\hline \multicolumn{5}{|c|}{ Short circuit } \\
\hline \multirow{5}{*}{$\mathrm{r}=1$} & 72.569 & 72.162 & 68.824 & 68.736 \\
\hline & 136.19 & 128.27 & 124.93 & 124.75 \\
\hline & 289.89 & 225.35 & 218.86 & 218.56 \\
\hline & 318.10 & 296.81 & 281.80 & 281.19 \\
\hline & 373.47 & 323.07 & 310.50 & 309.68 \\
\hline \multirow{5}{*}{$r=5$} & 154.14 & 154.86 & 153.62 & 153.99 \\
\hline & 183.73 & 186.08 & 183.57 & 183.67 \\
\hline & 544.47 & 530.31 & 528.10 & 528.67 \\
\hline & 580.13 & 585.25 & 572.11 & 573.17 \\
\hline & 716.01 & 716.42 & 705.46 & 706.46 \\
\hline \multirow{5}{*}{$r=20$} & 257.69 & 284.26 & 285.08 & 286.33 \\
\hline & 469.37 & 499.81 & 502.38 & 504.90 \\
\hline & 1253.2 & 1415.8 & 1401.4 & 1407.7 \\
\hline & 1521.9 & 1527.4 & 1526.1 & 1534.4 \\
\hline & 1555.9 & 1765.7 & 1757.5 & 1764.6 \\
\hline \multicolumn{5}{|c|}{ Open circuit } \\
\hline \multirow{5}{*}{$\mathrm{r}=1$} & 80.281 & 79.093 & 76.910 & 76.081 \\
\hline & 154.85 & 140.42 & 138.20 & 138.36 \\
\hline & 299.73 & 242.90 & 236.48 & 236.51 \\
\hline & 351.89 & 327.73 & 317.98 & 313.16 \\
\hline & 397.39 & 345.72 & 338.24 & 331.87 \\
\hline \multirow{5}{*}{$r=5$} & 162.34 & 161.03 & 161.91 & 160.26 \\
\hline & 198.07 & 199.98 & 197.90 & 198.16 \\
\hline & 552.07 & 539.90 & 538.66 & 539.29 \\
\hline & 629.27 & 627.02 & 622.81 & 615.36 \\
\hline & 763.78 & 754.82 & 754.54 & 745.80 \\
\hline \multirow{5}{*}{$r=20$} & 272.66 & 297.74 & 298.60 & 299.96 \\
\hline & 482.33 & 508.13 & 513.38 & 513.22 \\
\hline & 1340.7 & 1487.2 & 1473.6 & 1479.6 \\
\hline & 1541.7 & 1552.3 & 1545.6 & 1559.7 \\
\hline & 1629.9 & 1820.5 & 1825.2 & 1819.7 \\
\hline
\end{tabular}


Table 12. First five natural frequencies for the hemispherical sandwich shell with a hole.

\begin{tabular}{lcccc}
\hline & $\begin{array}{c}\text { C3D8E } \\
(200 \times 50 \times 3)\end{array}$ & $\begin{array}{c}\text { SHB8PSE } \\
(56 \times 14 \times 3)\end{array}$ & $\begin{array}{c}\text { C3D20E } \\
(60 \times 20 \times 3)\end{array}$ & $\begin{array}{c}\text { SHB20E } \\
(28 \times 7 \times 3)\end{array}$ \\
\hline Short circuit & & & & \\
\hline & 96.873 & 79.149 & 76.109 & 76.110 \\
& 193.18 & 118.04 & 114.60 & 113.80 \\
& 296.19 & 220.94 & 215.44 & 215.81 \\
& 363.22 & 301.63 & 289.37 & 289.86 \\
& 576.11 & 363.47 & 342.83 & 348.82 \\
\hline Open circuit & & & & \\
\hline & 97.953 & 80.407 & 77.366 & 77.447 \\
& 194.24 & 119.04 & 115.33 & 113.87 \\
& 299.25 & 221.51 & 217.30 & 215.88 \\
& 365.82 & 304.01 & 292.63 & 293.50 \\
& 581.14 & 364.21 & 346.70 & 348.95 \\
\hline
\end{tabular}




\section{Figures}

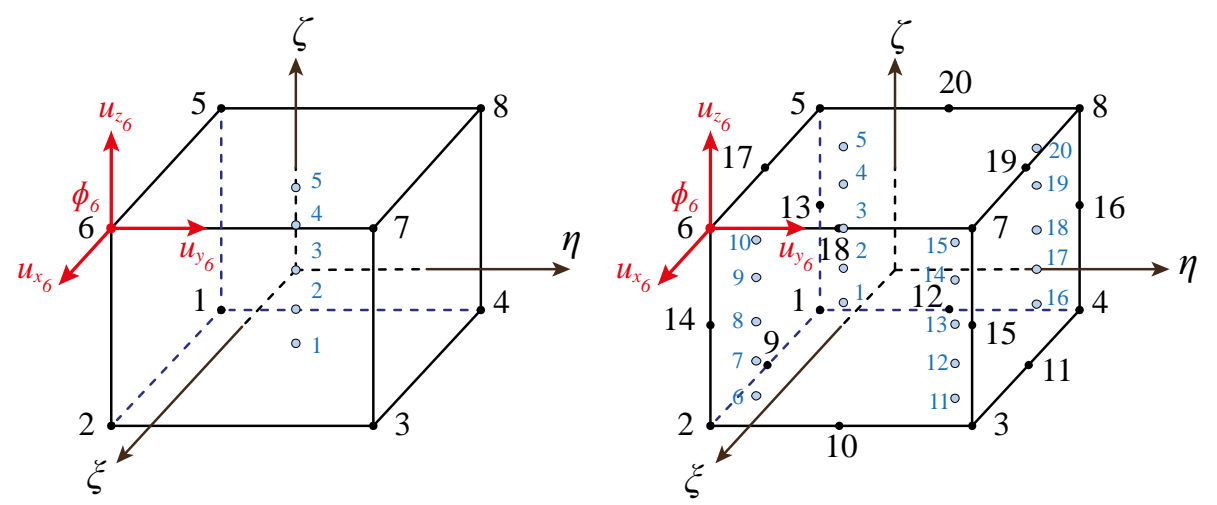

Figure 1. Schematic representation for the reference geometry of the SHB8PSE and SHB20E elements as well as for the location of their integration points in the case when the number of through-thickness integration points is $n_{\text {int }}=5$.

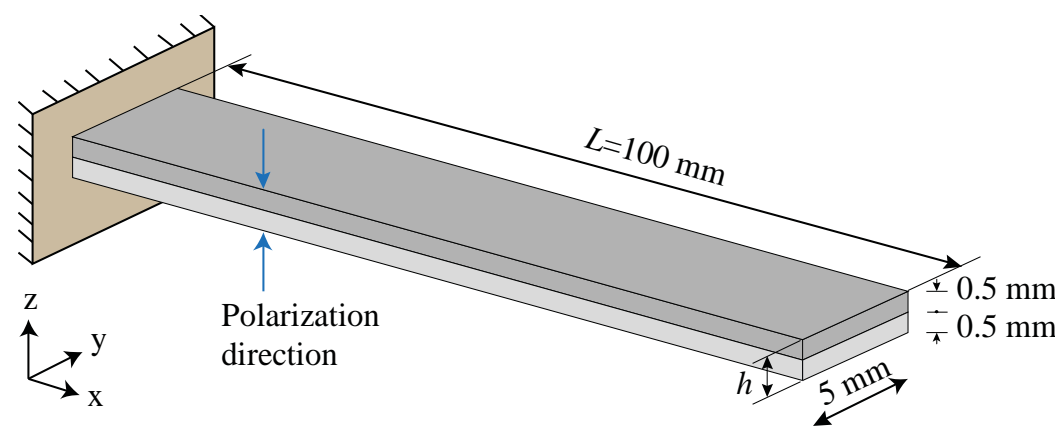

Figure 2. Cantilever bimorph beam. 


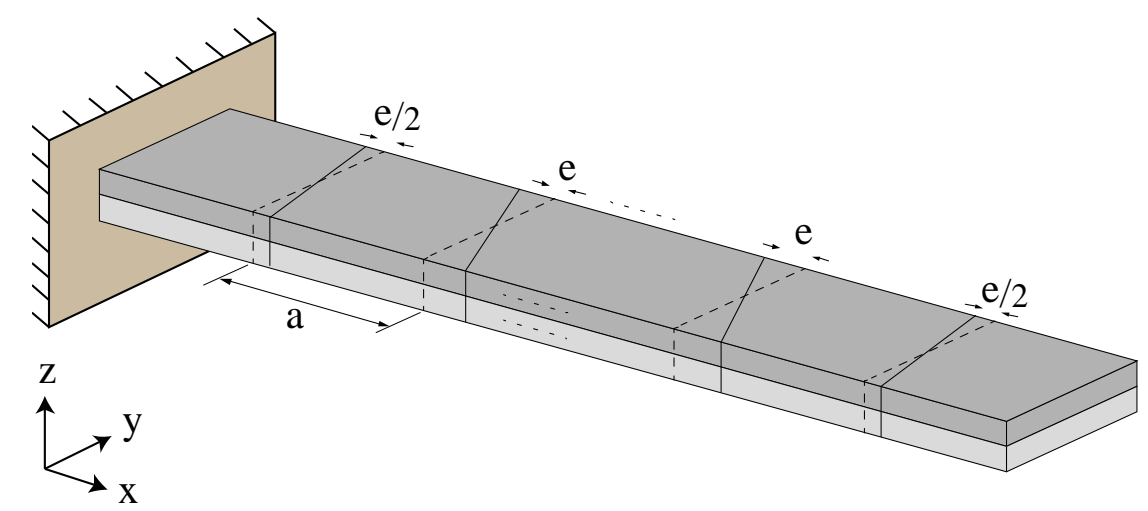

Figure 3. Cantilever bimorph beam with distorted mesh.

(a)

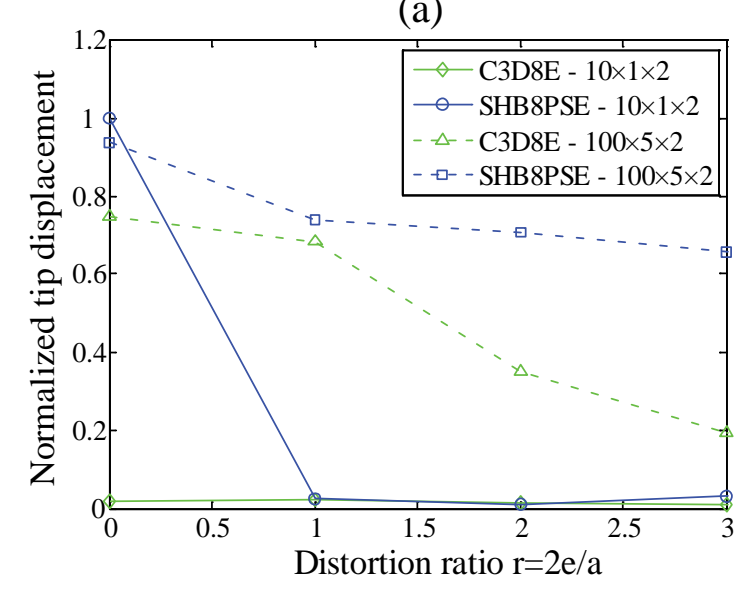

(b)

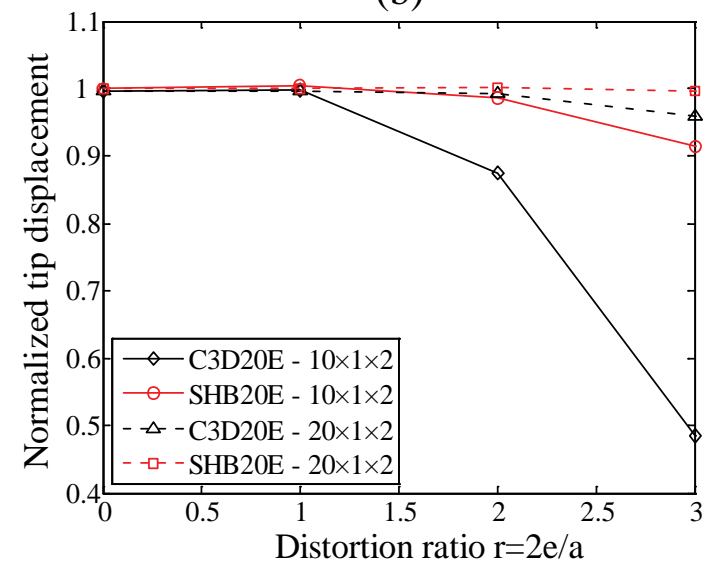

Figure 4. Convergence results for the cantilever bimorph beam problem in the case of distorted meshes; (a) linear hexahedral elements, (b) quadratic hexahedral elements. 


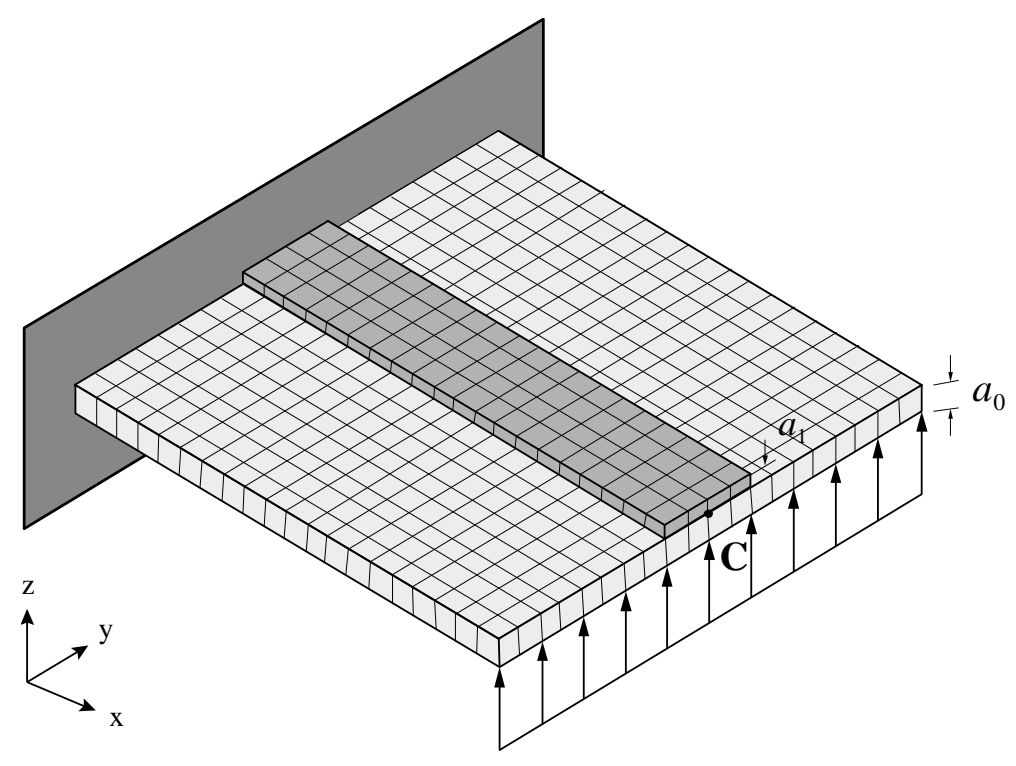

Figure 5. Model and discretization for the steel plate benchmark test with a piezoelectric sensor layer attached to its upper surface.
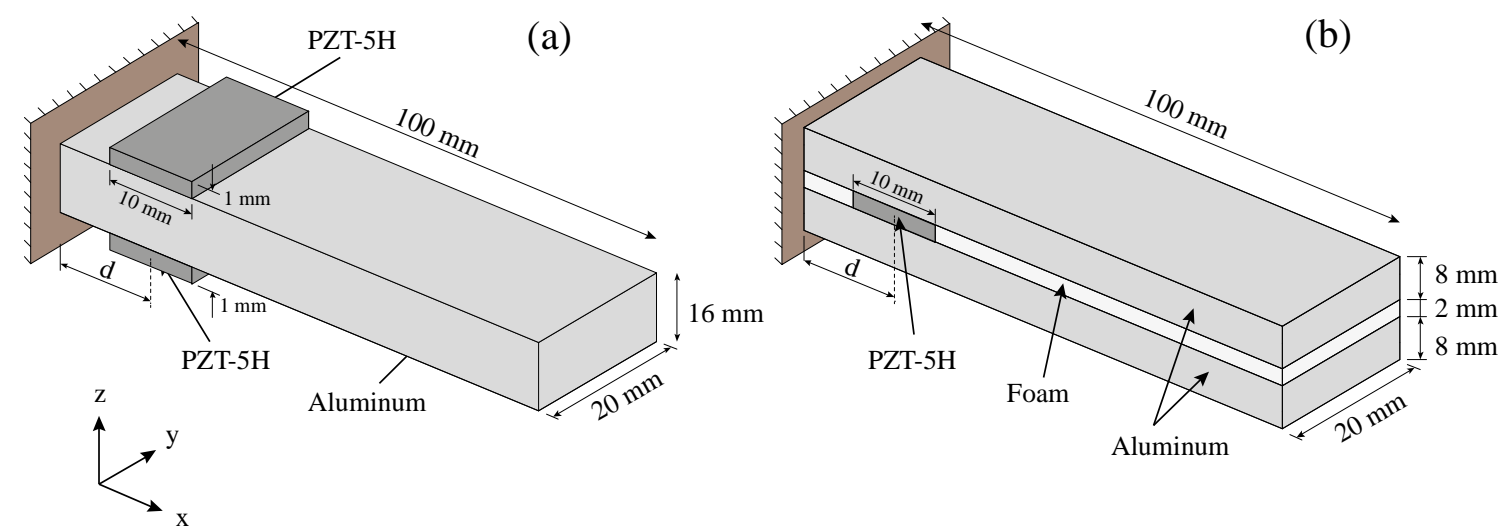

Figure 6. Cantilever sandwich beam, extension (a) and shear (b) actuation mechanisms. 

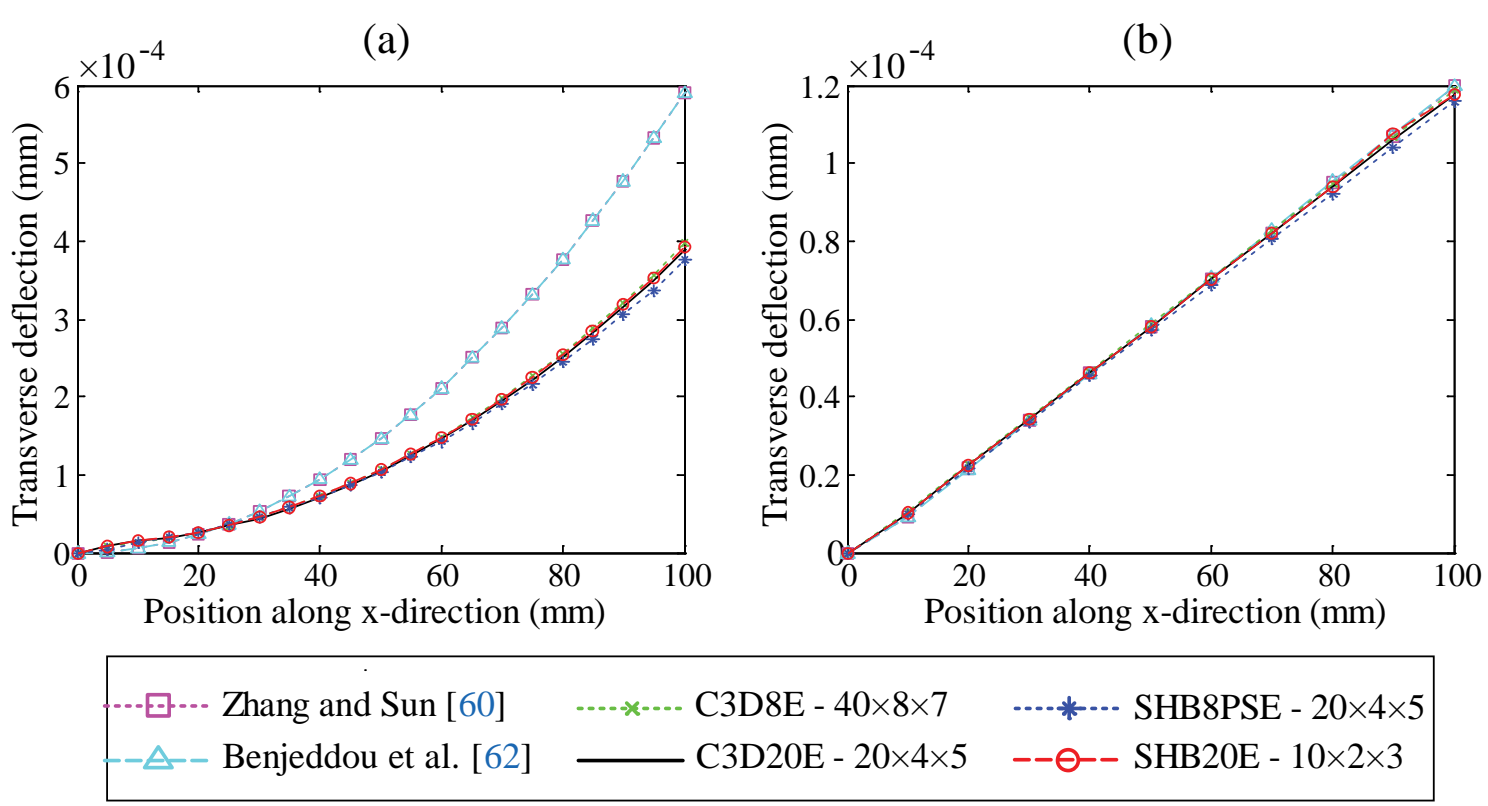

Figure 7. Transverse displacement of the beam for extension (a) and shear (b) mechanisms.
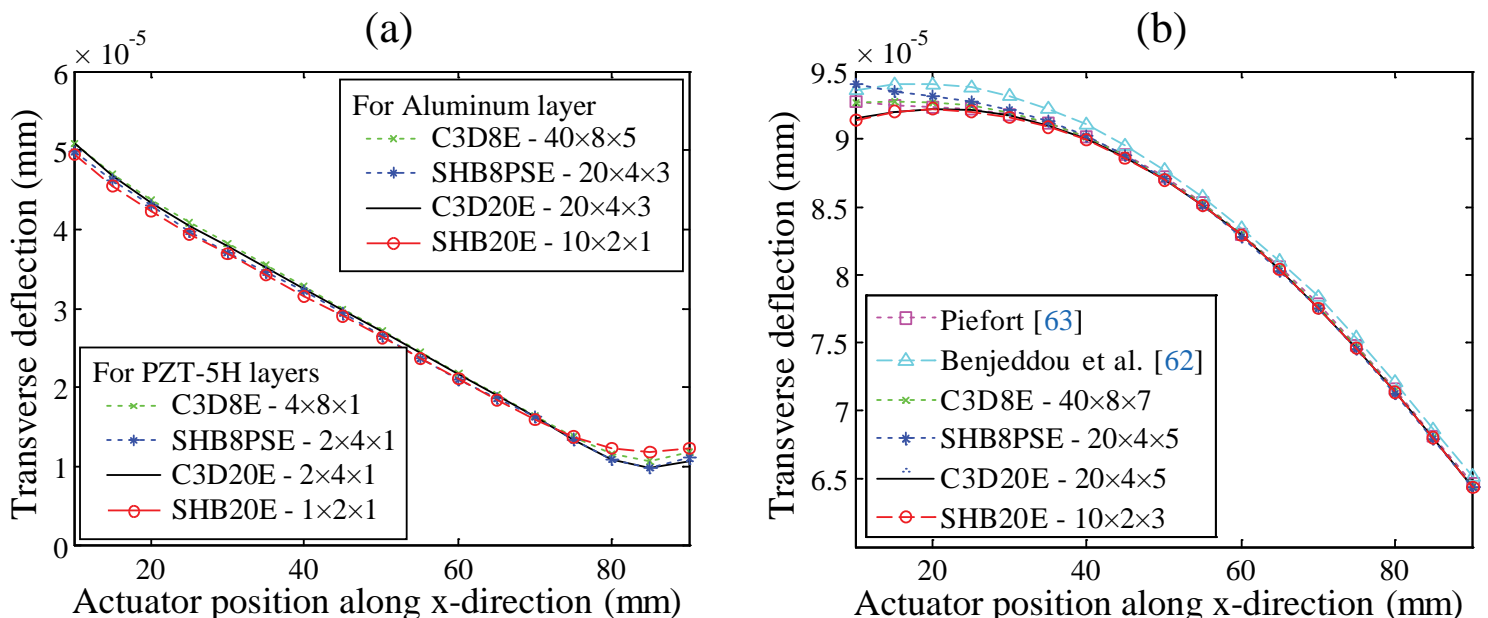

Figure 8. Tip displacement at the free edge of the beam for extension (a) and shear (b) mechanisms with different positions of piezoelectric patches. 


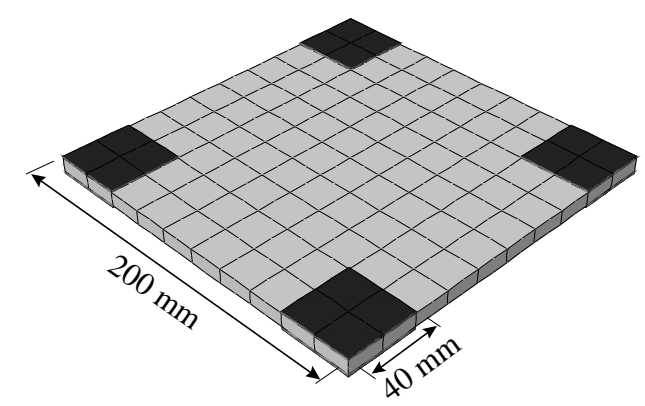

Configuration (a)

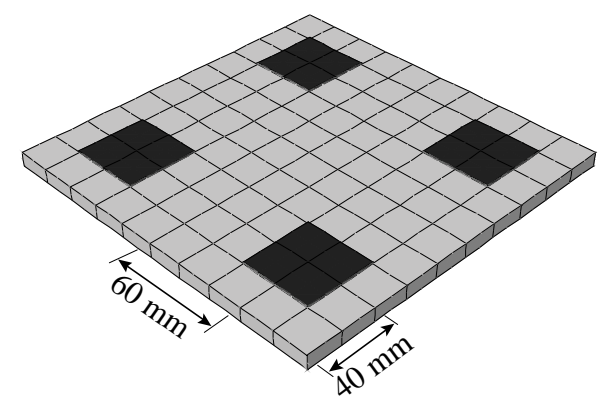

Configuration (c)

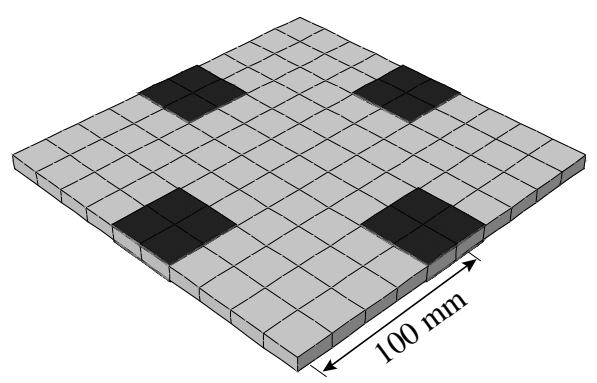

Configuration (b)

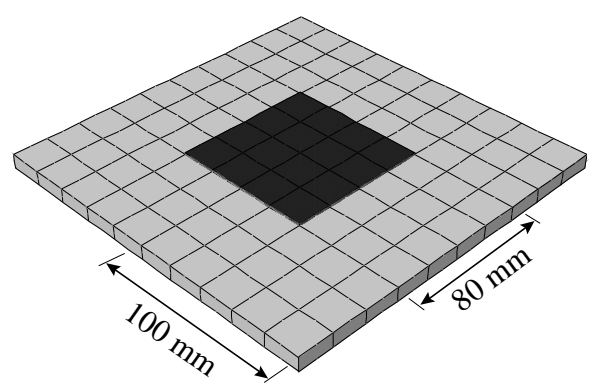

Configuration (d)

Figure 9. Square plate with piezoelectric patches. 
(a)

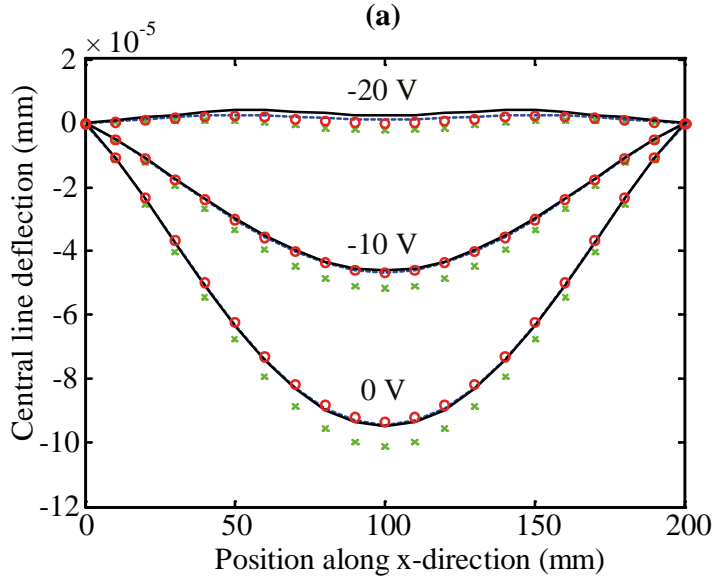

(c)

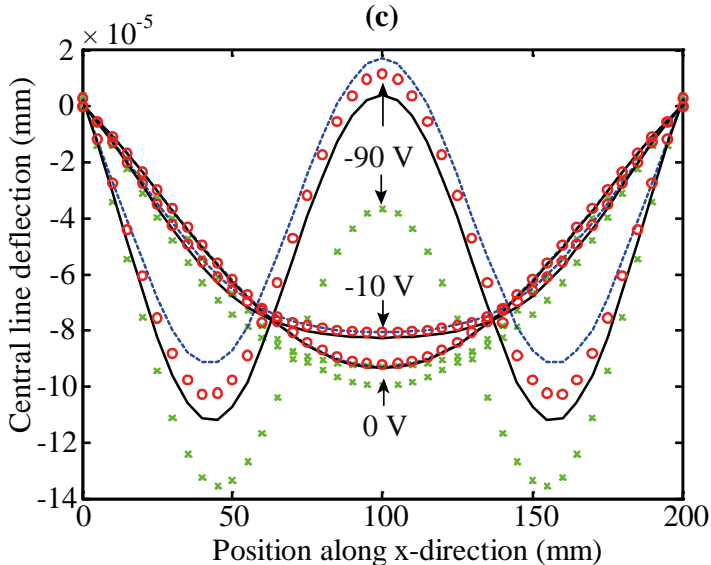

(b)

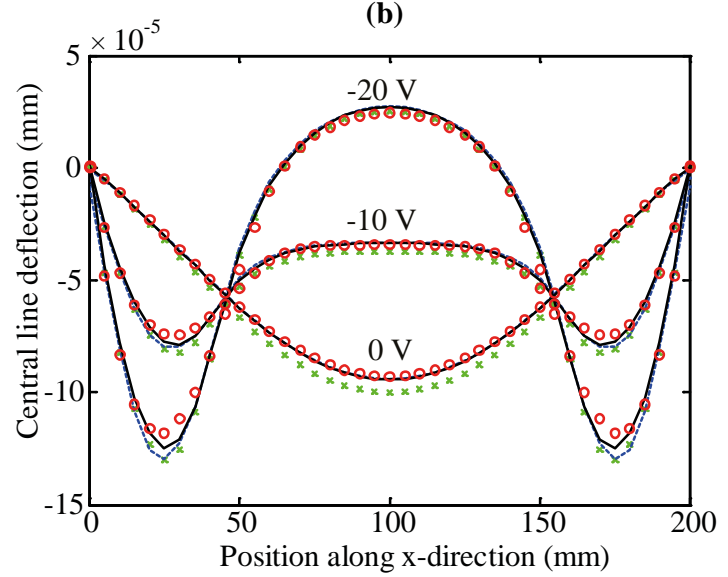

(d)

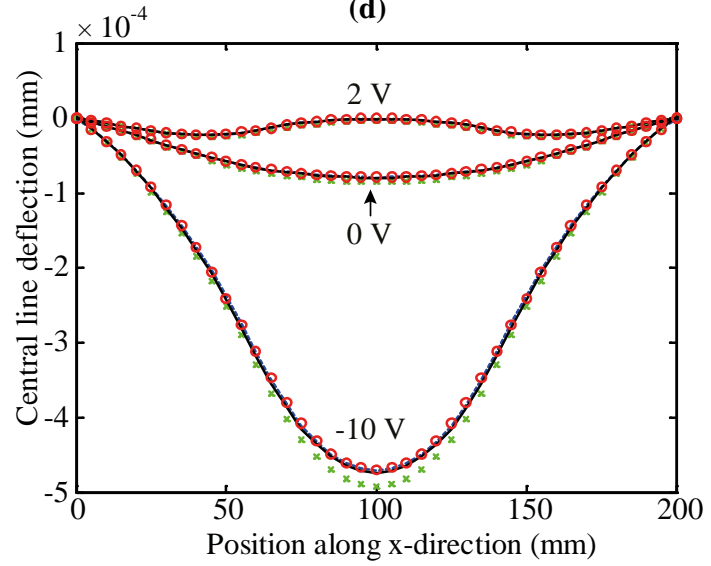

For Aluminum layer $\quad \times \quad \times \quad \times$ C3D $8-80 \times 80 \times 1$

SHB8PS - 20×20×1

For PZT-5H layers $\times \times$ C 3 D $8 E-16 \times 16 \times 1$ SHB8PSE $-4 \times 4 \times 1$
C3D20 - 20×20×1

$\circ \circ \circ$ SHB20 - 10×10×1

C3D20E - 4×4×1

Figure 10. Central line displacement for the square plate under uniform load and various values of voltage, for four different patch layouts. 


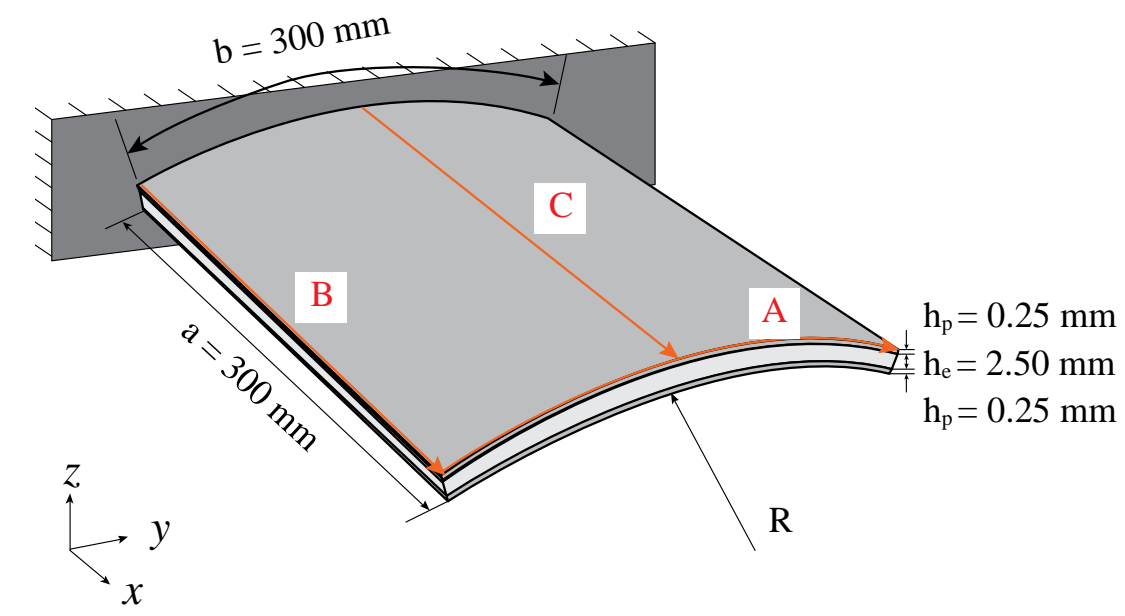

Figure 11. Cantilever curved sandwich shell.
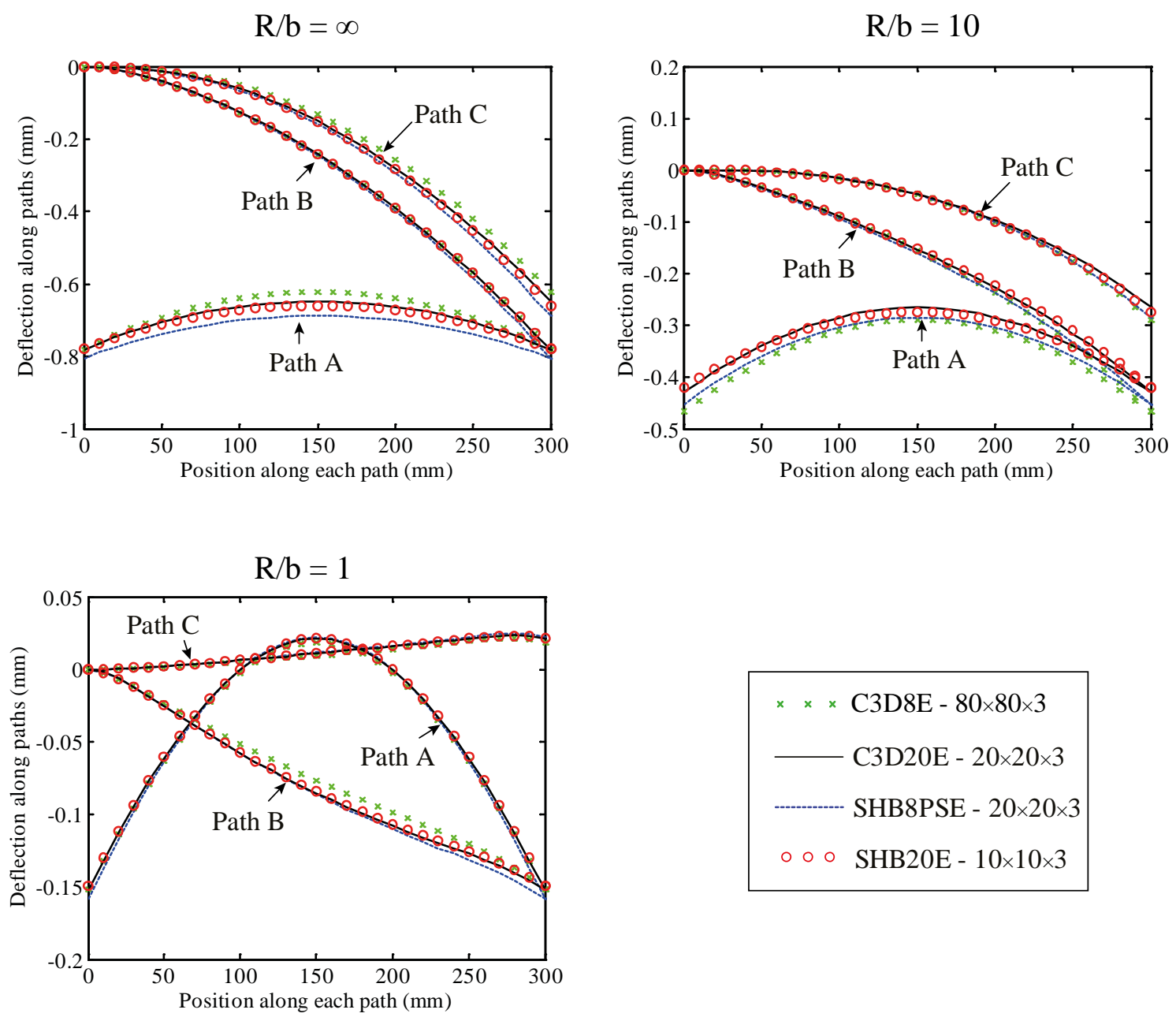

Figure 12. Deflection along paths A, B and C for the clamped curved sandwich shell, for different curvatures $R / b$. 


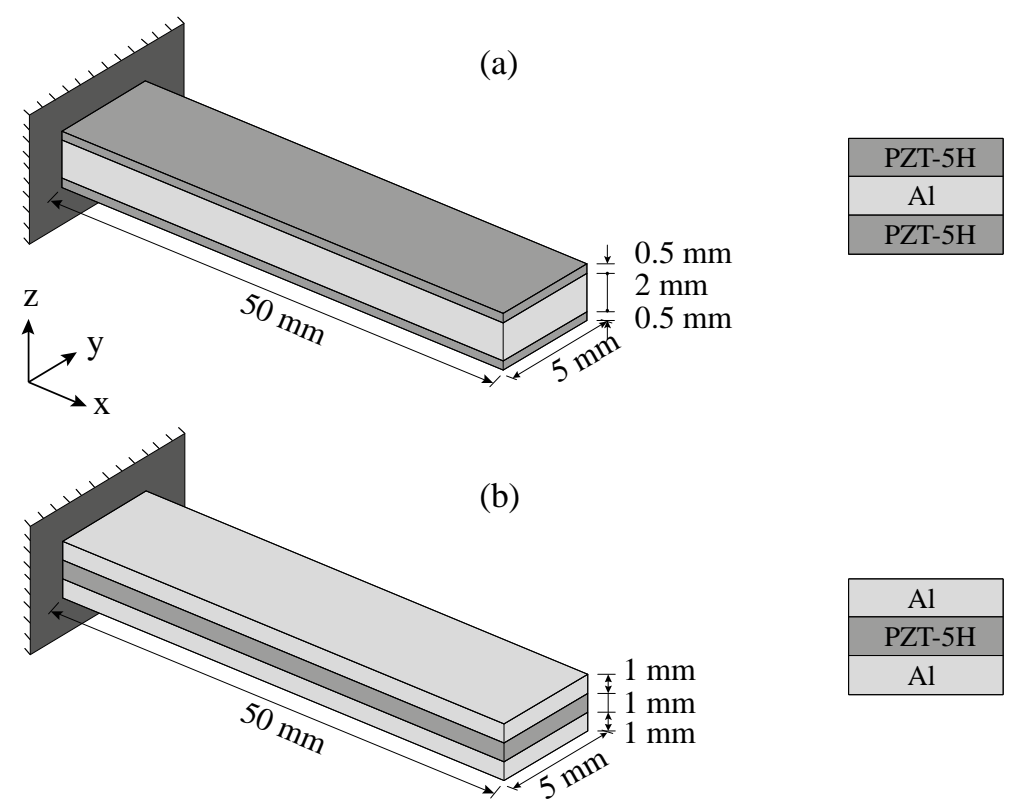

Figure 13. Cantilever sandwich beam, extension (a) and shear (b) actuation mechanisms.

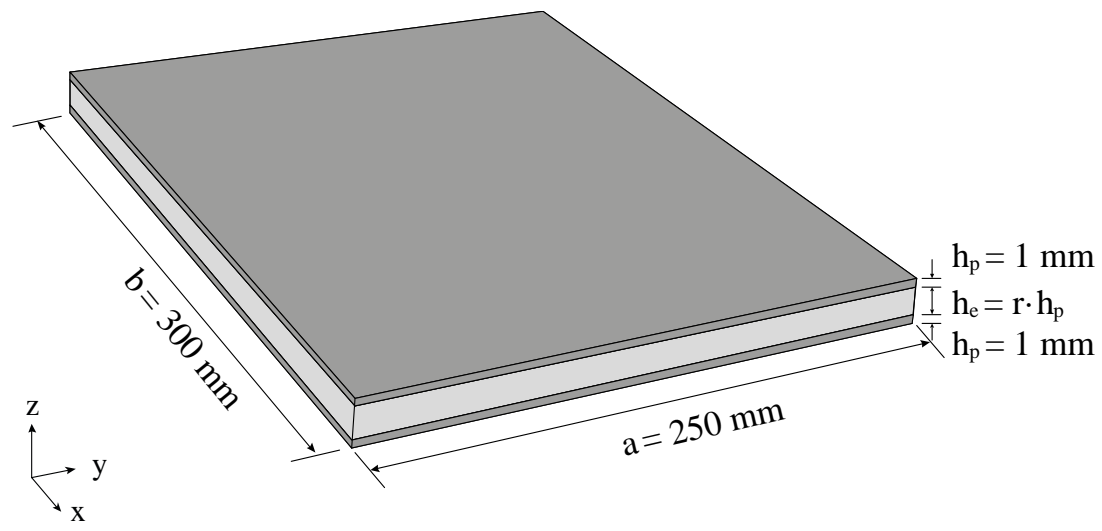

Figure 14. Simply supported rectangular sandwich plate. 


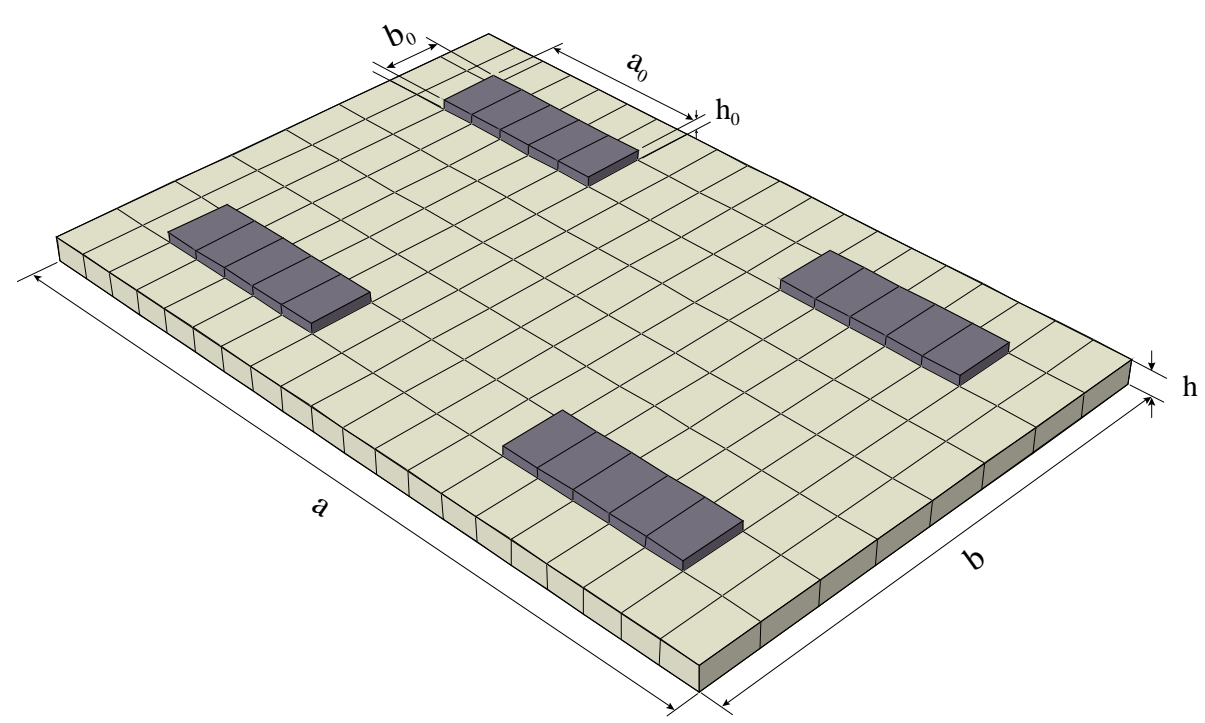

Figure 15. Cantilever plate with piezo-patches.

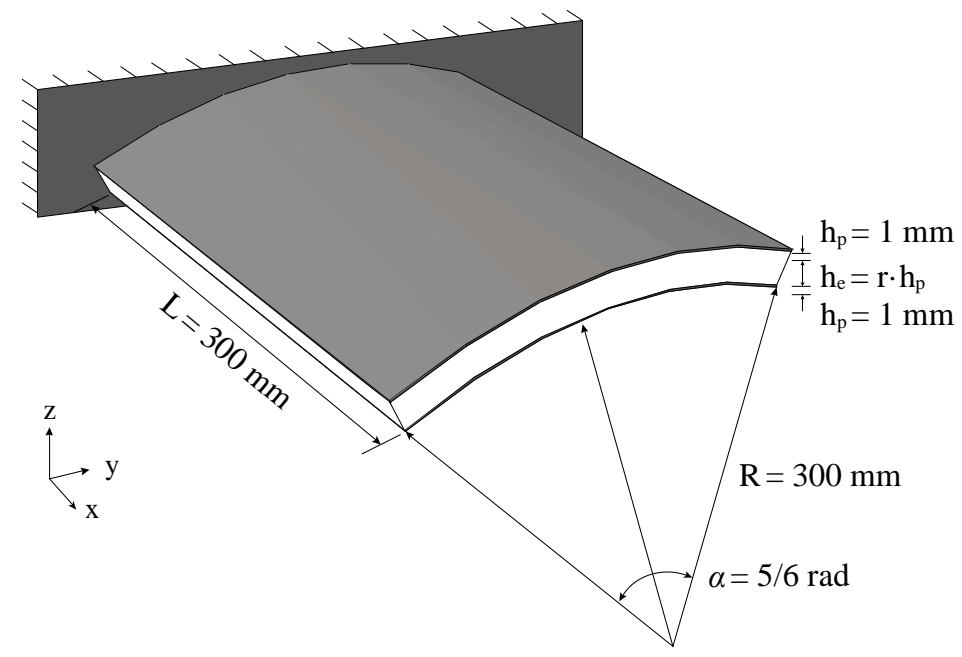

Figure 16. Curved cantilever sandwich blade. 


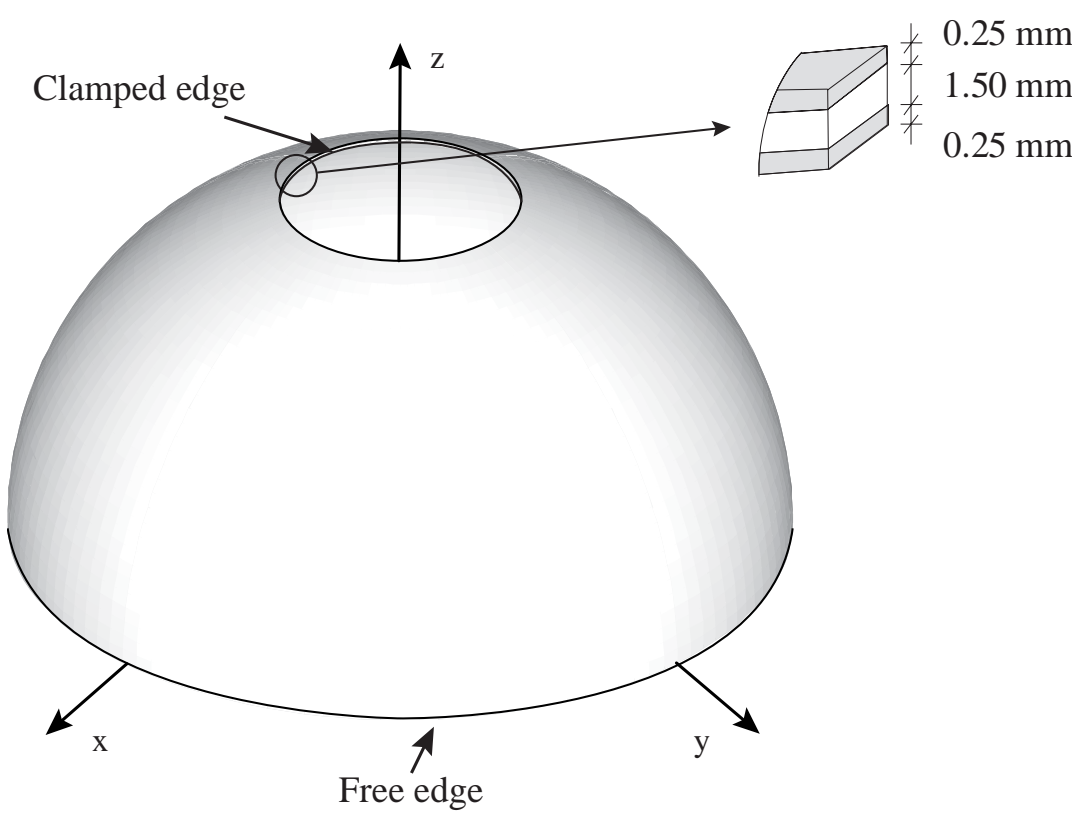

Figure 17. Hemispherical sandwich shell with a hole.
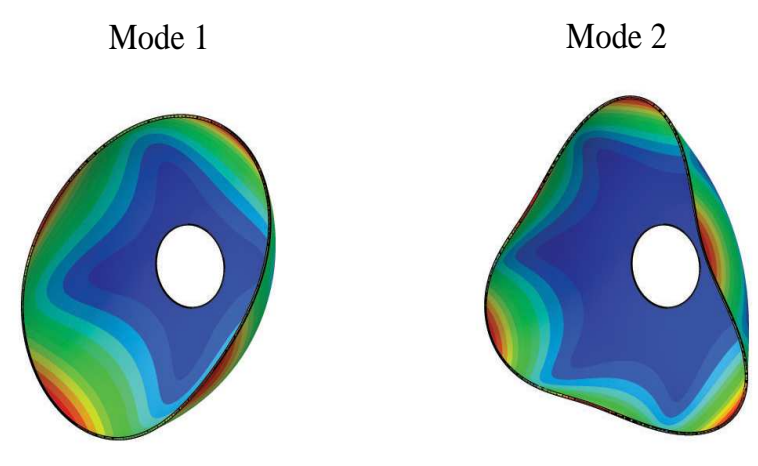

Mode 3
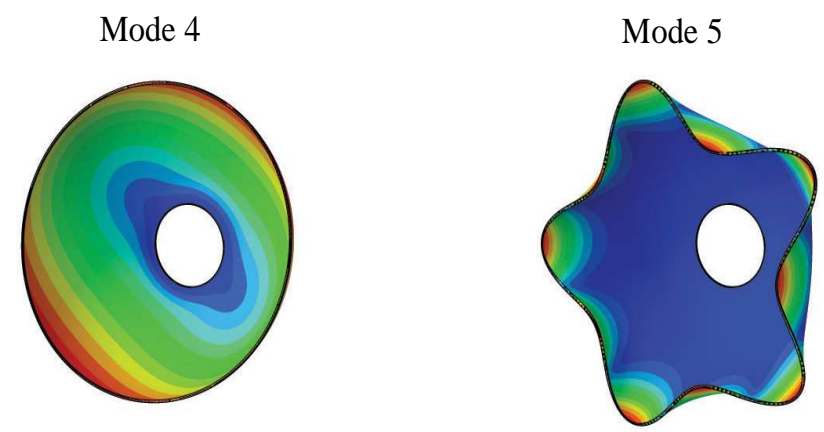

Figure 18. First five vibration modes for the hemispherical sandwich shell with a hole. 


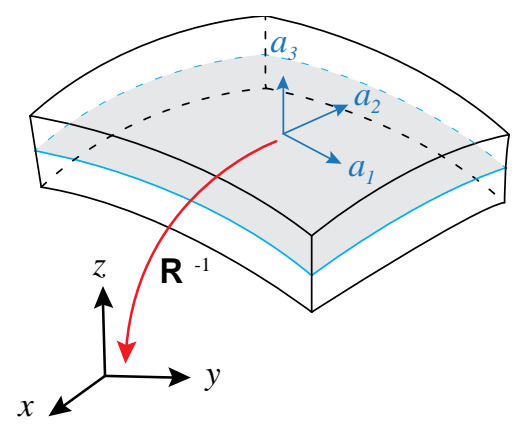

Figure C.1: Transformation from local physical frame to global frame. 